\title{
Radial velocity variability and stellar properties of FGK stars in the cores of NGC 2516 and NGC 2422
}

\author{
John I. Bailey, III, ${ }^{1 \star}$ Mario Mateo, ${ }^{2}$ Russel J. White, ${ }^{3}$ Stephen A. Shectman ${ }^{4}$ \\ and Jeffrey D. Crane ${ }^{4}$ \\ ${ }^{1}$ Leiden Observatory, Leiden University, PO Box 9513, NL-2300RA Leiden, The Netherlands \\ ${ }^{2}$ Department of Astronomy, University of Michigan, 1085 South University, Ann Arbor, MI 48109, USA \\ ${ }^{3}$ Department of Physics, and Astronomy, Georgia State University, PO Box 4106, Atlanta, GA 30302, USA \\ ${ }^{4}$ Carnegie Observatories, 813 Santa Barbara Street, Pasadena, CA 91101, USA
}

Accepted 2017 December 13. Received 2017 December 13; in original form 2016 September 12

\begin{abstract}
We present multi-epoch high-dispersion optical spectra obtained with the Michigan/Magellan Fibre System of 126 and 125 Sun-like stars in the young clusters NGC 2516 (141 Myr) and NGC 2422 (73 Myr). We determine stellar properties including radial velocity (RV), $T_{\text {eff }}$, $[\mathrm{Fe} / \mathrm{H}],[\alpha / \mathrm{Fe}]$ and the line-of-sight rotation rate, $v_{r} \sin (i)$, from these spectra. Our median RV precision of $80 \mathrm{~m} \mathrm{~s}^{-1}$ on individual epochs that span a temporal baseline of $1.1 \mathrm{yr}$ enables us to investigate membership and stellar binarity, and to search for sub-stellar companions. We determine membership probabilities and RV variability probabilities for our sample along with candidate companion orbital periods for a select subset of stars. In NGC 2516, we identified $81 \mathrm{RV}$ members, 27 spectroscopic binaries (17 previously identified as photometric binaries) and 16 other stars that show significant RV variability after accounting for average stellar jitter at the $74 \mathrm{~m} \mathrm{~s}^{-1}$ level. In NGC 2422, we identify 57 members, 11 spectroscopic binaries and three other stars that show significant RV variability after accounting for an average jitter of $138 \mathrm{~m} \mathrm{~s}^{-1}$. We use Monte Carlo simulations to verify our stellar jitter measurements, determine the proportion of exoplanets and stellar companions to which we are sensitive, and estimate companion-mass limits for our targets. We also report mean cluster metallicity, velocity and velocity dispersion based on our member targets. We identify 58 non-member stars as RV variables, 24 of which have RV amplitudes that imply stellar or brown-dwarf mass companions. Finally, we note the discovery of a separate RV clustering of stars in our NGC 2422 sample.
\end{abstract}

Key words: techniques: radial velocities-techniques: spectroscopic-planets and satellites: detection-stars: abundances - binaries: spectroscopic-open clusters and associations: individual: (NGC 2516, NGC 2422).

\section{INTRODUCTION}

Exoplanets found in open stellar clusters have significant potential to inform our understanding of planetary formation and system evolution (e.g. Paulson, Cochran \& Hatzes 2004; Quinn et al. 2012, 2014; Meibom et al. 2013; Howell et al. 2014; Brucalassi et al. 2014; Bailey et al. 2016). Their appeal stems from the well-known ages and well-characterized environments offered by open clusters. This allows planets found therein generally to offer greater leverage on the relative importance that core accretion (Mizuno et al. 1980) and disc gravitational instabilities (Boss 1997) play in formation and the various migration mechanisms play in the evolution

^E-mail: baileyji@umich.edu of so-called hot Jupiters (e.g. disc coupling, Goldreich \& Tremaine 1980; Lin, Bodenheimer \& Richardson 1996; dynamical scattering, Rasio \& Ford 1996; Jurić \& Tremaine 2008; or even secular interactions, Fabrycky \& Tremaine 2007). Unfortunately, the number of exoplanets confirmed at present in clusters remains small $(<10)$, despite the considerable effort expended in finding such systems. This reflects the difficulty of surveying large numbers of stars in clusters to sufficiently high velocity precision suitable for the detection of exoplanets.

In Bailey et al. (2016, hereafter B16), we introduced a new approach to obtain highly multiplexed radial velocities (RVs) with sufficient precision to detect warm and hot Jupiters around solar analogues in open cluster out to $\sim 1 \mathrm{kpc}$ using the Michigan/Magellan Fibre System (M2FS; Mateo et al. 2012). We showed that we are able to measure RVs for up to 128 stars over a half-degree 
Table 1. Pointings.

\begin{tabular}{|c|c|c|c|c|c|c|c|c|c|c|}
\hline Cluster & Messier & $\begin{array}{c}\text { RA } \\
(2000)\end{array}$ & $\begin{array}{l}\text { Dec. } \\
(2000)\end{array}$ & $\begin{array}{l}\text { Age } \\
(\mathrm{Myr})\end{array}$ & $\begin{array}{l}\text { Distance } \\
\quad(\mathrm{pc})\end{array}$ & $E(B-V)$ & $N_{\text {epoch }}$ & $N_{\text {targ }}$ & V & $B-V$ \\
\hline NGC 2516 & - & $7: 58: 42$ & $-60: 46: 36$ & 141 & 346 & 0.11 & 12 & 126 & $11.68-15.09$ & $0.46-1.26$ \\
\hline NGC 2422 & M47 & $7: 36: 30$ & $-14: 29: 42$ & 73 & 491 & 0.07 & 10 & 125 & $12.20-16.10$ & $0.45-1.43$ \\
\hline
\end{tabular}

Note. The coordinates listed correspond to our field centres and, although near, are not at the cluster centre. Both distances as well as the reddening for NGC 2422 are from Kharchenko et al. (2005). Target photometry is from J01 (NGC 2516) and P03/UCAC4 (NGC 2422). The reddening for NGC 2516 is from Sung et al. (2002). The age for NGC 2516 is from Meynet, Mermilliod \& Maeder (1993) and for NGC 2422 from Loktin, Gerasimenko \& Malysheva (2001). Note that Kharchenko et al. (2005) gives ages of 120 and $132 \mathrm{Myr}$, albeit with errors of $\sim 70 \mathrm{Myr}$.

field of view with a measurement precision of $25 \mathrm{~m} \mathrm{~s}^{-1}$ for sufficiently bright, slowly rotating $\left(\lesssim 10 \mathrm{~km} \mathrm{~s}^{-1}\right)$, Sun-like stars and to $45-65 \mathrm{~m} \mathrm{~s}^{-1}$ at magnitudes typical for such stars in open clusters within $1 \mathrm{kpc}$. We also showed that we are able to obtain precise measurements of $T_{\text {eff }},[\mathrm{Fe} / \mathrm{H}],[\alpha / \mathrm{Fe}]$ and $v_{r} \sin (i)$. Our technique, thus, allows us to search efficiently for warm and hot Jupiters in open clusters with ages ranging from about 100 Myr to nearly $1 \mathrm{Gyr}$ while simultaneously characterizing their host environment well.

As a first test of our technique, we carried out a survey of all the Sun-like stars in the cores of the 141-Myr-old open cluster NGC 2516 and 73-Myr-old cluster NGC 2422, the youngest open clusters yet surveyed using RV techniques. Here we report the first results of our survey: effective temperatures $\left(T_{\text {eff }}\right)$, iron and light element abundances $([\mathrm{Fe} / \mathrm{H}]$ and $[\alpha / \mathrm{Fe}]$ from template fitting), projected rotational velocity $\left[v_{r} \sin (i)\right]$, mean radial velocity $(\overline{\mathrm{RV}})$ and observed RV standard deviation $\left(\sigma_{\mathrm{obs}}\right)$ measurements for the 251 stars in our sample, 126 in NGC 2516 and 125 in NGC 2422. We also report RV-based membership probabilities and cluster properties (e.g. velocity dispersion, binary fraction and abundance) and examine the level of stellar jitter in each cluster. Finally, we identify all stars in our sample that exhibit statistically significant RV variability, reporting a number of spectroscopic binaries and identifying a small number of potential exoplanet hosts that merit further investigation.

In Section 2, we review our observational programme and the details of the specific stars we target in NGC 2516 and NGC 2422. Then, in Section 3, we review our analysis methodology and present stellar properties for our targets. Section 4 describes our approach to spectroscopic binaries in our sample and the Monte Carlo simulations we used to investigate a number of questions related to binarity, membership and stellar jitter later in this text. We use Section 5 to detail our RV membership approach, describe a small number of notable stars, report our findings for both NGC 2516 and NGC 2422, and also note the presence of a separate association of stars contaminating our NGC 2422 sample. Section 6 looks at the cluster RV, RV dispersion and the projected rotation and metallicity of cluster members. Here we consider the RV dispersion, binary fraction and abundances of both clusters. We also compare the cluster $v_{r} \sin (i)$ distributions with that of the Pleiades. In Section 7, we investigate the level of stellar jitter in our targets and determine an average level for each cluster. Finally, Section 8 presents the results of our RV variability analysis. We cover our companion-mass limits and report a number of RV variables - including several with significant periodicities indicative of stellar or sub-stellar companions meriting a prompt follow-up.

\section{STELLAR SAMPLE AND OBSERVATIONS}

We selected Sun-like stars in the 141-Myr- and 73-Myr-old open clusters NGC 2516 and NGC 2422 as targets for our study. These clusters are within $500 \mathrm{pc}$. They are rich in solar analogues and have approximately solar metallicity. They both have recent photometric membership catalogues that are photometrically complete for selecting Sun-like targets (Jeffries, Thurston \& Hambly 2001; Prisinzano et al. 2003, hereafter J01 and P03), and they have angular sizes and sky densities that are well matched to the 128 fibres M2FS can deploy across its half-degree field of view. Table $1-$ from B16 - provides the coordinates, colour and magnitude ranges, and number of epochs obtained for our pointings in each cluster along with cluster age, distance and reddening.

In NGC 2516, we selected targets that J01 identified as photometric single (79) or binary (47) members with colours and magnitudes consistent with F5V-K5V spectral types in our field of view, which was also constrained by our need for a bright central star for use as a Shack-Hartman reference. This sample of 126 stars was then cross-matched with the UCAC4 catalogue (Zacharias et al. 2013) to extract astrometry. In NGC 2422, we used the same approach to select photometric members (100) from the P03 catalogue, which does not distinguish between single and binary members. Due to the smaller number of P03 targets, we expanded our selection out in colour from the MS defined by P03 members using the UCAC4 catalogue until we had sufficient targets to fill the available fibres, selecting an additional 25 stars in our adopted pointing. We stress that with the available fibres, we are able to target every star in each half-degree field that could plausibly be a solar-analogue member.

We refer to the total sample of 126 stars in NGC 2516 and the 100 stars from P03 in NGC 2422 as photometric members in this paper and the additional 25 targets in NGC 2422 as candidate members. The photometric properties and positions of both the parent samples and final observed samples are illustrated in Figs 1 and 2, which show colour-magnitude diagrams and sky charts for NGC 2516 and NGC 2422. Tables A1 and A2, provided only as machine-readable tables online and at the CDS, list target IDs, coordinates, literature photometry, number of usable epochs ( signal to noise $\mathrm{S} / \mathrm{N}>12$ ) and the mean per-pixel $\mathrm{S} / \mathrm{N}$ for each target. They also report numerous other results that will be described in later sections. Table 2 lists the contents of these tables and provides references to the pertinent sections of the text while Table 3 provides an abbreviated example of Table A1.

As described in B16, we also targeted six stars with similar RAs from the Gaia RVS catalogue (Soubiran et al. 2013) for use as RV standards and to test our stellar property analysis. A summary of these stars is given in table 3 of B16.

We observed our targets using M2FS, a multi-object fibre-fed spectrograph located on the Magellan/Clay 6.5-m telescope at Las Campanas Observatory in Chile. M2FS was employed in crossdispersed echelle mode with $45-\mu \mathrm{m}$ fibre slits and the Hot Jupiter filter to obtain $\sim 130$ - $\AA$-wide spectra centred at $7230 \AA$ with a median resolving power of 50000 ( $R$ varies slightly with fibre, wavelength and focus) and a median per-pixel $\mathrm{S} / \mathrm{N}$ of 50 for all 251 of our targets. This wavelength region was selected for its optimal combination of telluric and stellar absorption lines, the former of 

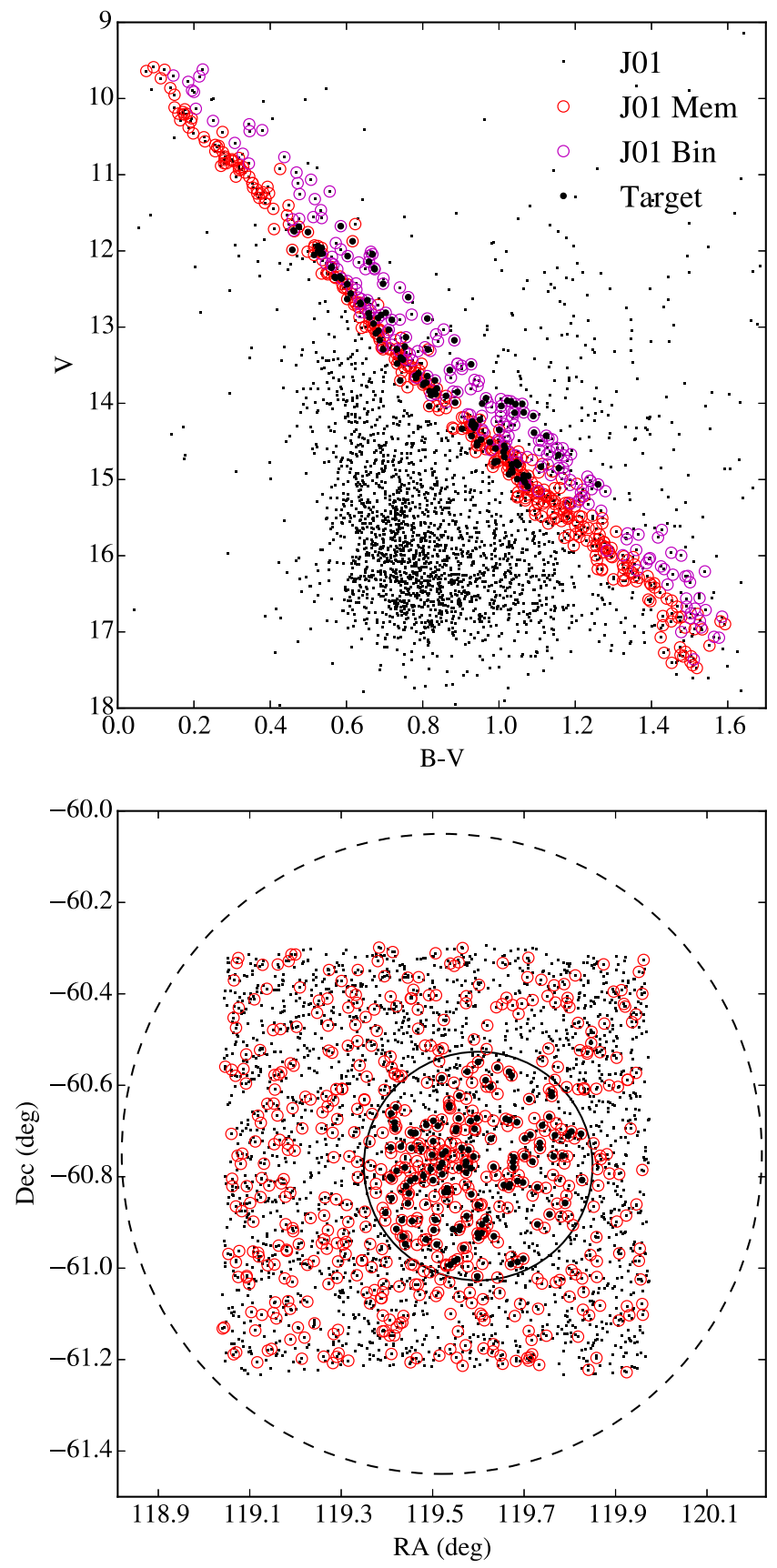

Figure 1. Colour-magnitude diagram and sky plot of our pointing in NGC 2516. Upper: Stars in the J01 catalogue as minuscule black points with stars flagged as photometric single members circled in red or photometric member binaries in purple. Stars we targeted are shown as large black marks. Lower: NGC 2516 stars with all photometric members (single or binary) circled in red. The square is the CCD footprint used by J01. The dashed black circle represents the cluster's nominal radius as reported in Kharchenko et al. (2005) and the solid black circle the M2FS field of view around our pointing centre.

which our modelling process uses as a simultaneous measure of both the wavelength-to-pixel mapping and the instrument point-spread function. Further details of the M2FS configuration we used, our rationale for this wavelength region and data reduction procedure are provided in $\mathrm{B} 16$.

The dates, number of stars targeted, median per-pixel $\mathrm{S} / \mathrm{N}$ and total exposure times (typically from three or four back-to-back ex-
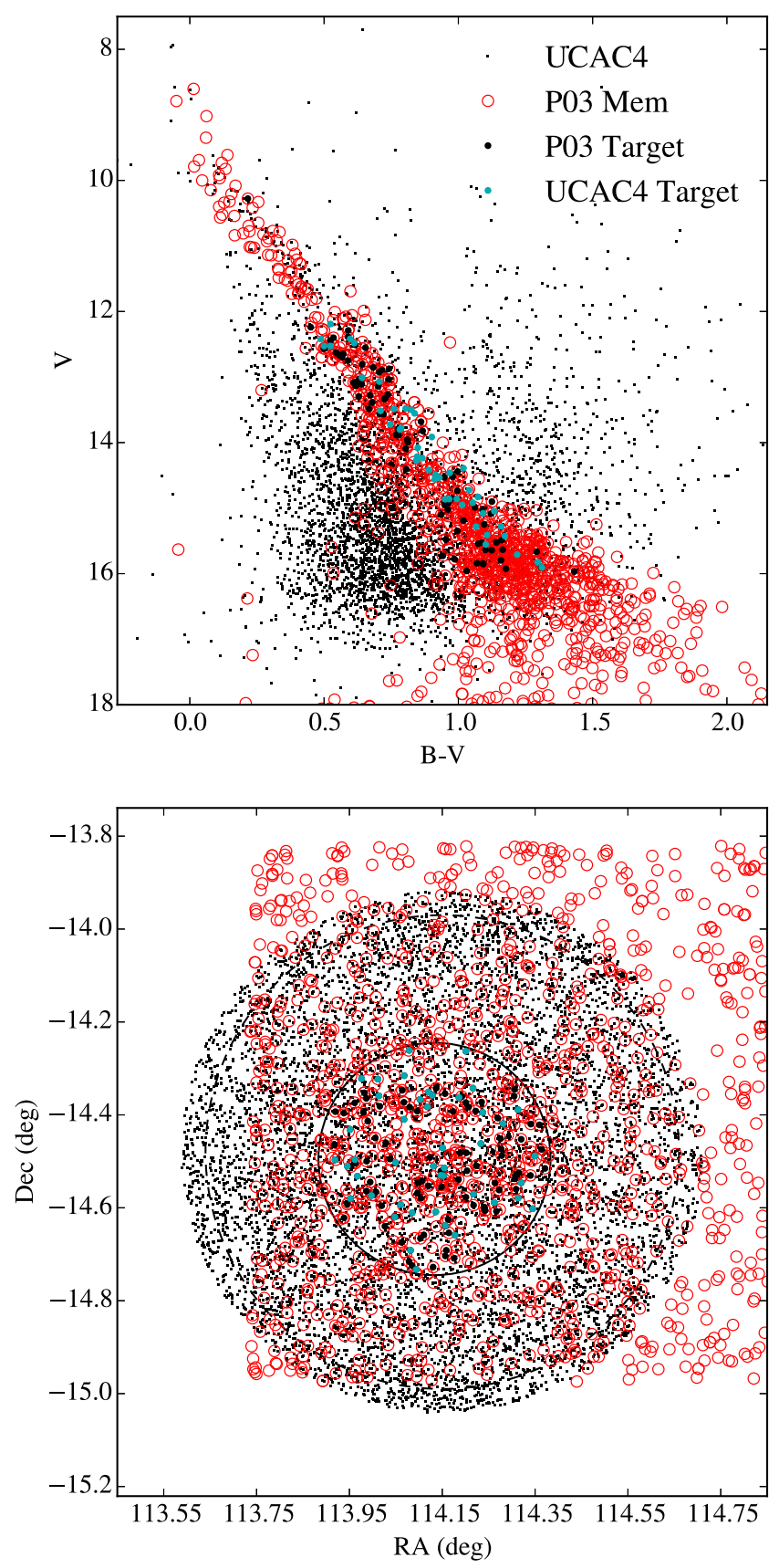

Figure 2. Colour-magnitude diagram and sky plot of our pointings in NGC 2422. Upper: Stars in the UCAC4 catalogue within 1.1 cluster radii of the centre of NGC 2422. Stars in the P03 catalogue (which includes only photometric members) are circled in red. Our targets are shown as large black marks if from P03 or cyan marks if from UCAC4. Lower: NGC 2422 stars with photometric members circled in red. The square is the CCD footprint used by $\mathrm{P} 03$. The dashed black circle represents the cluster's nominal radius as reported in Kharchenko et al. (2005) and the solid black circle the M2FS field of view around our pointing centre.

posures) for each of our epochs are listed in Table 4. Exposure times ranged from $1.7 \mathrm{~h}$ to $3.1 \mathrm{~h}$ and median per-pixel S/N from 28 to 65 . In two epochs, operational issues resulted in eight and 23 stars not being targeted. Over the course of our campaign, an evolving set of damaged or dead fibres impacted our ability to obtain spectra of various targets. This, along with a wide magnitude range and variable seeing, resulted in a number of targets for which some (in 
Table 2. Columns in online Tables A1 (NGC 2516) and A2 (NGC 2422).

\begin{tabular}{|c|c|c|c|}
\hline Column & Name & Unit & Description \\
\hline 1 & ID & - & UCAC4 ID \\
\hline 2 & LitID & - & J01 (NGC 2516) or P03 (NGC 2422) ID (if extant) \\
\hline 3 & RAdeg & deg & Right ascension from UCAC4 \\
\hline 4 & DEdeg & $\operatorname{deg}$ & Declination from UCAC4 \\
\hline 5 & Vmag & mag & $V$ magnitude \\
\hline 6 & $\mathrm{~B}-\mathrm{V}$ & mag & $B-V$ colour \\
\hline 7 & PSrc & - & Photometry source: J01 (NGC 2516), P03 (NGC 2422) or U (UCAC4) \\
\hline 8 & $\mathrm{~N}$ & - & Number of usable spectra obtained \\
\hline 9 & $\mathrm{~S} / \mathrm{N}$ & - & Mean S/N (per pixel) \\
\hline 10 & Teff & $\mathrm{K}$ & Adopted effective temperature, including correction for members (Section 3.2) \\
\hline 11 & e+_Teff & $\mathrm{K}$ & Upper $1 \sigma$ uncertainty on $T_{\text {eff }}$ \\
\hline 12 & e-_Teff & $\mathrm{K}$ & Lower $1 \sigma$ uncertainty on $T_{\text {eff }}$ \\
\hline 13 & f_Teff & - & {$[0,1] T_{\text {eff }}$ correction applied } \\
\hline 14 & {$[\mathrm{Fe} / \mathrm{H}]$} & dex & Adopted $[\mathrm{Fe} / \mathrm{H}]$ abundance (Section 3.2) \\
\hline 15 & $\mathrm{e}+[\mathrm{Fe} / \mathrm{H}]$ & dex & Upper $1 \sigma$ uncertainty on $[\mathrm{Fe} / \mathrm{H}]$ \\
\hline 16 & $\mathrm{e}-[\mathrm{Fe} / \mathrm{H}]$ & $\operatorname{dex}$ & Lower $1 \sigma$ uncertainty on $[\mathrm{Fe} / \mathrm{H}]$ \\
\hline 17 & {$[\mathrm{a} / \mathrm{Fe}]$} & dex & Adopted $\alpha$-element abundance (Section 3.2) \\
\hline 18 & $\mathrm{e}+\_[\mathrm{a} / \mathrm{Fe}]$ & dex & Upper $1 \sigma$ uncertainty on $[\alpha / \mathrm{Fe}]$ \\
\hline 19 & $\mathrm{e}-\_[\mathrm{a} / \mathrm{Fe}]$ & dex & Lower $1 \sigma$ uncertainty on $[\alpha / \mathrm{Fe}]$ \\
\hline 20 & vrot & $\mathrm{km} \mathrm{s}^{-1}$ & Adopted line-of-sight stellar rotational velocity (Section 3.2) \\
\hline 21 & e+_vrot & $\mathrm{km} \mathrm{s}^{-1}$ & Upper $1 \sigma$ uncertainty on $v_{r} \sin (i)$ \\
\hline 22 & e-_vrot & $\mathrm{km} \mathrm{s}^{-1}$ & Lower $1 \sigma$ uncertainty on $v_{r} \sin (i)$ \\
\hline 23 & $\log (g)$ & - & $\log (g)$ adopted for fitting (Section 3.2) \\
\hline 24 & RVel & $\mathrm{m} \mathrm{s}^{-1}$ & Weighted mean barycentric RV (Section 3.2) \\
\hline 25 & e_RVel & $\mathrm{m} \mathrm{s}^{-1}$ & Bootstrapped error on the weighted mean barycentric RV \\
\hline 26 & sig_obs & $\mathrm{m} \mathrm{s}^{-1}$ & $\sigma_{\mathrm{obs}}$, error weighted standard deviation of measured RVs (Section 3.2) \\
\hline 27 & sig_meas & $\mathrm{m} \mathrm{s}^{-1}$ & $\sigma_{\text {meas }}$, mean RV measurement error (Section 3.2) \\
\hline 28 & Pmem & - & $P_{\mathrm{RV}}, \mathrm{RV}$ membership probability (Section 5) \\
\hline 29 & Mem & - & Membership flag: member $(\mathrm{M})$, non-member $(\mathrm{N})$, probable member $(\mathrm{P})$, no-data $(\mathrm{X})$ (Section 5) \\
\hline 30 & Type & - & RV single (S), RV binary (B), double-lined binary (SB2), continuum (C), no-data (X) (Section 4, Section 5.1) \\
\hline 31 & sig_jit & $\mathrm{m} \mathrm{s}^{-1}$ & Adopted stellar jitter, $\sigma_{\text {jitter }}($ Section 7) \\
\hline 32 & Pvar & - & Probability that target is an RV variable (Section 8) \\
\hline 33 & Pvar_jit & - & Probability that target is an RV variable in the presence of adopted stellar jitter (Section 8) \\
\hline 34 & Period & days & Optimized value of most significant periodogram peak above 95 per cent confidence interval (Section 8.1) \\
\hline 35 & M3 & $\mathrm{M}_{\text {Jup }}$ & 95 per cent companion-mass limit at $3 \mathrm{~d}$ (Section 8.2$)$ \\
\hline 36 & M10 & $\mathrm{M}_{\mathrm{Jup}}$ & 95 per cent companion-mass limit at $10 \mathrm{~d}$ (Section 8.2) \\
\hline 37 & M20 & $\mathrm{M}_{\mathrm{Jup}}$ & 95 per cent companion-mass limit at $20 \mathrm{~d}$ (Section 8.2) \\
\hline 38 & Mstar & $\mathrm{M}_{\odot}$ & Stellar mass adopted for companion detectability test (Section 8.2) \\
\hline
\end{tabular}

Table 3. Example of online Table A1: Properties of targets in NGC 2516.

\begin{tabular}{|c|c|c|c|c|c|c|c|c|c|c|c|c|c|c|}
\hline ID & $\begin{array}{c}\text { Literature } \\
\text { ID }\end{array}$ & $\begin{array}{c}\text { RA } \\
\text { (deg) }\end{array}$ & $\begin{array}{l}\text { Dec. } \\
\text { (deg) }\end{array}$ & $\begin{array}{c}V \\
(\mathrm{mag})\end{array}$ & $\begin{array}{l}B-V \\
(\mathrm{mag})\end{array}$ & PSrc & $N$ & $\overline{\mathrm{S} / \mathrm{N}}$ & $\begin{array}{l}T_{\text {eff }} \\
(\mathrm{K})\end{array}$ & $\mathrm{f} \_T_{\text {eff }}$ & $\begin{array}{c}{[\mathrm{Fe} / \mathrm{H}]} \\
(\mathrm{dex})\end{array}$ & $\begin{array}{c}{[\alpha / \mathrm{Fe}]} \\
(\mathrm{dex})\end{array}$ & $\begin{array}{c}v_{r} \sin (i) \\
\left(\mathrm{km} \mathrm{s}^{-1}\right)\end{array}$ & $\log (g)$ \\
\hline $147-012265$ & 7864 & 119.50997 & -60.77981 & 12.07 & 0.603 & J01 & 11 & 115 & $6447 \pm 50$ & 1 & $-0.25 \pm 0.04$ & $0.04_{-0.02}^{+0.01}$ & $16.9 \pm 0.5$ & 4.4 \\
\hline $147-012424$ & 11307 & 119.89213 & -60.71620 & 13.70 & 0.741 & J01 & 12 & 54 & $6116_{-33}^{+35}$ & 1 & $-0.09 \pm 0.02$ & $0.03_{-0.02}^{+0.01}$ & $6.4 \pm 0.2$ & 4.5 \\
\hline $146-012601$ & 11233 & 119.88376 & -60.81251 & 13.88 & 0.834 & J01 & 10 & 39 & $5239 \pm 19$ & 1 & $-0.29 \pm 0.02$ & $0.12 \pm 0.01$ & $16.1 \pm 0.2$ & 4.6 \\
\hline $147-012249$ & 7590 & 119.48121 & -60.72243 & 13.99 & 0.885 & J01 & 12 & 51 & $5372_{-13}^{+17}$ & 1 & $-0.14 \pm 0.01$ & $0.06 \pm 0.01$ & $6.7 \pm 0.1$ & 4.6 \\
\hline 147-012499 & 12874 & 120.08582 & -60.71043 & 13.49 & 0.927 & J01 & 12 & 51 & $5480 \pm 79$ & 1 & $-0.36 \pm 0.10$ & $0.06 \pm 0.01$ & $8.4 \pm 1.3$ & 4.5 \\
\hline $\begin{array}{l}\mathrm{RV} \\
\left(\mathrm{m} \mathrm{s}^{-1}\right) \\
\end{array}$ & $\begin{array}{c}\sigma_{\mathrm{obs}} \\
\left(\mathrm{m} \mathrm{s}^{-1}\right)\end{array}$ & $\begin{array}{c}\sigma_{\text {meas }} \\
\left(\mathrm{m} \mathrm{s}^{-1}\right)\end{array}$ & $\mathrm{P}_{\mathrm{mem}}$ & Mem. & Type & $\begin{array}{c}\sigma_{\mathrm{jit}} \\
\left(\mathrm{m} \mathrm{s}^{-1}\right)\end{array}$ & $P_{\mathrm{var}}$ & $P_{\text {var, jit }}$ & $\begin{array}{c}\text { Period } \\
\text { (day) }\end{array}$ & $\begin{array}{c}M_{3} \\
\left(\mathrm{M}_{\text {Jup }}\right) \\
\end{array}$ & $\begin{array}{c}M_{10} \\
\left(\mathrm{M}_{\text {Jup }}\right)\end{array}$ & $\begin{array}{c}M_{20} \\
\left(\mathrm{M}_{\text {Jup }}\right)\end{array}$ & $\begin{array}{c}M_{\text {star }} \\
\left(\mathrm{M}_{\odot}\right)\end{array}$ & \\
\hline $37677 \pm 11052$ & 35789 & 196 & 0.00 & $\mathrm{P}$ & SB2 & 74 & 1.00 & 1.00 & - & 7.9 & 12.2 & 23.9 & 1.17 & \\
\hline $15450 \pm 6734$ & 23160 & 75 & 0.00 & $\mathrm{P}$ & SB2 & 74 & 1.00 & 1.00 & 30.0 & 3.7 & 5.6 & 11.3 & 1.11 & \\
\hline $31158 \pm 6325$ & 19304 & 159 & 0.00 & $\mathrm{P}$ & B & 74 & 1.00 & 1.00 & 1.9 & 5.8 & 9.0 & 21.8 & 0.82 & \\
\hline $25997 \pm 4535$ & 16386 & 62 & 0.86 & M & B & 74 & 1.00 & 1.00 & 23.2 & 3.0 & 4.5 & 9.2 & 0.89 & \\
\hline $18343 \pm 4125$ & 14814 & 87 & 0.00 & $\mathrm{P}$ & SB2 & 74 & 1.00 & 1.00 & 78.7 & 3.5 & 5.4 & 10.8 & 0.87 & \\
\hline
\end{tabular}

Note. Tables A1 and A2 are available online in machine-readable format. Table 2 provides a description of the columns in the full table. 
Table 4. Cluster observations.

\begin{tabular}{lccc}
\hline Date & $N$ & $\begin{array}{c}\text { Median } \\
\text { S/N }\end{array}$ & $\begin{array}{c}\text { Exposure time } \\
(\mathrm{s})\end{array}$ \\
\hline & \multicolumn{2}{c}{ NGC 2516 } & \\
2013 November 22 & 118 & 57 & 6600 \\
2013 November 24 & 126 & 28 & 6000 \\
2013 November 27 & 126 & 38 & 6300 \\
2013 November 28 & 126 & 59 & 7200 \\
2013 November 30 & 126 & 44 & 6800 \\
2014 February 16 & 103 & 46 & 9600 \\
2014 February 17 & 126 & 65 & 9000 \\
2014 February 21 & 126 & 57 & 9000 \\
2014 December 9 & 126 & 48 & 7200 \\
2014 December 10 & 126 & 33 & 7200 \\
2014 December 11 & 126 & 63 & 9000 \\
2014 December 12 & 126 & 55 & 7200 \\
& & & \\
2013 December 1 & 125 & 60 & 7200 \\
2014 February 18 & 125 & 55 & 9000 \\
2014 February 19 & 124 & 46 & 7200 \\
2014 February 22 & 124 & 41 & 7200 \\
2014 February 26 & 125 & 38 & 9000 \\
2014 December 12 & 125 & 35 & 9000 \\
2014 December 13 & 125 & 51 & 10150 \\
2014 December 17 & 125 & 71 & 9800 \\
2014 December 20 & 125 & 57 & 11200 \\
2014 December 22 & 125 & 59 & 10800 \\
\hline
\end{tabular}

one case, all) of our spectra fell below the $\mathrm{S} / \mathrm{N}$ limit (12) at which we are able to run our analysis reliably (see Section 3 ).

\section{SPECTROSCOPICALLY MEASURED STELLAR PROPERTIES}

\subsection{Analysis}

Here we review the key methods and performance of our analysis procedure. Full details can be found in B16. The exposures obtained with M2FS were individually bias-corrected and treated with the L.A. Cosmic algorithm (van Dokkum 2001) to detect and flag cosmic ray hits. We did not flat-field our data due to limitations in the M2FS flat-field system. Frames were then summed, correcting for both cosmic ray hits and scattered light. The spectra in each frame were traced and extracted to 1D using PyRAF APALL. Throughout this process, we maintained a variance frame and performed an identical extraction on it to obtain a variance spectrum, which we use for determining the mean per-pixel $\mathrm{S} / \mathrm{N}$ and in the fitting process to weight each pixel.

We fitted a model of each spectrum built from a telluric transmission template (Wallace et al. 2011) and a synthetic spectrum interpolated from the PHOENIX grid (Husser et al. 2013) to each of our spectra and adopted the mean of the best-fitting values of $T_{\text {eff }},[\mathrm{Fe} / \mathrm{H}],[\alpha / \mathrm{Fe}]$ and $v_{r} \sin (i)$ as our values for each star. When fitting, we use

$\log (g)=\log \left(\frac{9.44 \times 10^{9}}{\left(T_{\text {eff }} / \mathrm{K}\right)^{16 / 11}}\right)$,

which is derived from the mass-luminosity, mass-radius and temperature-luminosity relations, to compute values for $\log (g)$ that are sufficiently accurate. The resulting $\log (g)$ values are likely representative of the true values for member stars in NGC 2516 and NGC 2422. We caution that the values we report are not measurements of $\log (g)$ and stress that our results are not appreciably affected by the sub half-dex error this approximation may cause. Additional details and the derivation of equation (1) are in section 4.1 .1 of $\mathrm{B} 16$.

RVs were determined in a final iteration of fits in which stellar properties are held fixed at their adopted values as described above. The exact iterative process was determined by optimizing our prescription to minimize the observed RV dispersion $\left(23 \mathrm{~m} \mathrm{~s}^{-1}\right)$ of our RV standard HIP 48331, a well-studied K5V star $(V=7.7)$ from the Gaia-RVS catalogue (Soubiran et al. 2013).

We also used the HIP 48331 data to predict the RV precision as a function of $\mathrm{S} / \mathrm{N}$. This was done by artificially degrading the 35 spectra of HIP 48331 to several specific lower $\mathrm{S} / \mathrm{N}$ values and repeating the spectroscopic fitting analysis on each set. We then fitted a line to the ratio of the standard deviation of RVs ( $\left.\sigma_{\text {obs }}\right)$ over the range of $\mathrm{S} / \mathrm{N}$ values to the photometric uncertainty estimate as derived in Butler et al. (1996) to determine an empirically motivated correction to that relation, which we then use to determine the RV precision for each individual spectrum. The determined precisions range from $\sim 25 \mathrm{~m} \mathrm{~s}^{-1}$ at very high $\mathrm{S} / \mathrm{N}$ values, consistent with the above, to $\sim 100 \mathrm{~m} \mathrm{~s}^{-1}$ at $\mathrm{S} / \mathrm{N}$ of about 15 .

We further verified the predicted precision using 60 unstacked spectra of bright science targets from individual nights. As described in section 5.2.1 'Sky Emission' of B16, each epoch typically consists of three to five subsequently obtained spectra with S/N of 15-45 for targets with spectral type $\sim$ K3-F5. Though these science targets may be affected by stellar jitter that biases the RV measurements, we expected this to introduce a constant systematic offset over the duration of these spectra and not increase the RV dispersion. We found good agreement with the relation determined using HIP 48331, though we saw some evidence that our preferred fitting approach is biased to the initial RV for stars with $v_{r} \sin (i) \gtrsim$ $30 \mathrm{~km} \mathrm{~s}^{-1}$. We caution that our individual RV measurement errors may be underestimated by a factor of 2-4 in these cases, increasing with $v_{r} \sin (i)$, though note that this is a conservative bias (e.g. decreasing the likelihood of a false positive). While cross-correlating to obtain an initial RV estimate often alleviates the false minimum found by the optimizer, we suspect that a Bayesian analysis of our model would show the non-linear least-squares optimizer underreports the errors in these cases. Due to the long Markov chain Monte Carlo (MCMC) run times, we have not yet performed this analysis.

Individual RV measurements (e.g. from each epoch) have a measurement error $\left(\sigma_{\text {meas }}\right)$ that results from each fit and is computed using the empirically derived correction to the photometric uncertainly derived in Butler et al. (1996), which we determined using our observations of the RV standard HIP 48331 (see above). The uncertainty is, principally, a function of the $\mathrm{S} / \mathrm{N}$ at each pixel and the slope of the line profiles in each fitted model. Further details and verification of our measurement errors can be found in section 5.2.1 of B16. This approach yields errors of more than $100 \mathrm{~m} \mathrm{~s}^{-1}$ at our pipeline S/N limit of 12 to a systematic limit of $\sim 25 \mathrm{~m} \mathrm{~s}^{-1}$ at $\mathrm{S} / \mathrm{N}$ levels above 200 for slowly rotating stars (see fig. 14 in B16).

\subsection{Spectroscopic results}

We report $T_{\text {eff }},[\mathrm{Fe} / \mathrm{H}],[\alpha / \mathrm{Fe}], v_{r} \sin (i), \mathrm{RV}, \sigma_{\text {obs }}, \sigma_{\text {meas }}$ and the associated errors for our targets in Tables A1 and A2. The statistical uncertainties of the four stellar parameters were determined in B16 by looking at the distribution of best-fitting values relative to their multi-epoch means grouped into $\mathrm{F}, \mathrm{G}$ and $\mathrm{K}$ bins. We found $T_{\text {eff }},[\mathrm{Fe} / \mathrm{H}]$ and $[\alpha / \mathrm{Fe}]$, and $v_{r} \sin (i)$ to have typical single-epoch 

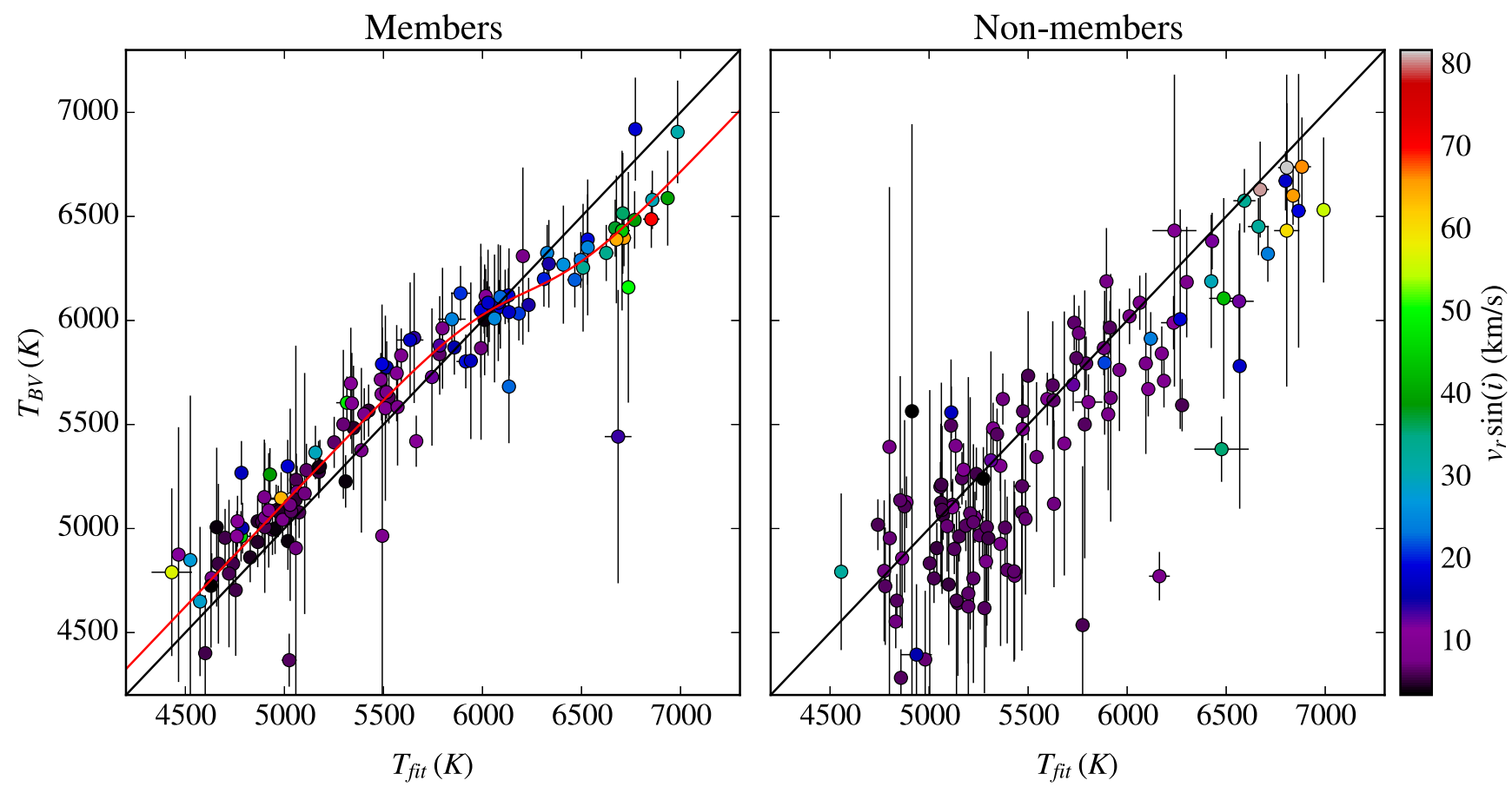

Figure 3. $T_{\mathrm{eff}}$ versus $T_{B-V}$ for the combined cluster sample. Left: Values for stars with $P_{\mathrm{RV}} \geq 50$ per cent (Section 5) plotted with coloured points indicating $v_{r} \sin (i)$. The red line represents our correction to Casagrande's scale given in equation (2). Note that $\Delta(B-V)$ of 0.05 corresponds to $\Delta T \sim 100 \mathrm{~K}$ here, about the level at which many members are offset below $6200 \mathrm{~K}$, and perhaps suggesting a correction to $E(B-V)$. Right: Targets with $P_{\mathrm{RV}}<50$ per cent. Here, it seems the inapplicable reddening values for non-members enters into play. In both panels, the diagonal black line shows equivalence as a guide to the eye. Errors on $T_{B-V}$ are dominated by photometric errors while errors on $T_{\text {eff }}$ are as reported in this text.

precisions of $75 \mathrm{~K}, 0.05 \mathrm{dex}$ and $0.75 \mathrm{~km} \mathrm{~s}^{-1}$ with a slight dependence on spectral type. The errors reported for $T_{\text {eff }},[\mathrm{Fe} / \mathrm{H}],[\alpha / \mathrm{Fe}]$ and $v_{r} \sin (i)$ in Tables $\mathrm{A} 1$ and $\mathrm{A} 2$ are from table 7 of $\mathrm{B} 16$ corrected for the total number of epochs of each target (i.e. divided by $\sqrt{N}$ ) or the target's standard error, whichever is greater. Typical precisions are $\pm 30 \mathrm{~K}, \pm 0.02 \mathrm{dex}$ and $\pm 0.3 \mathrm{~km} \mathrm{~s}^{-1}$, exclusive of systematic effects, which are considered later in this section.

The systemic RVs are the inverse variance weighted $\left(\sigma_{\text {meas }}^{-2}\right)$ means of our individual barycentre-corrected values. The errors on the means were determined by a Gaussian process resampling bootstrap and are typically around $40 \mathrm{~m} \mathrm{~s}^{-1}$. In section 5.2 of B16, we found our measurements had an offset of $74 \pm 72 \mathrm{~m} \mathrm{~s}^{-1}$ relative to the RV scale of Soubiran et al. (2013) when using five RV standards spanning the F5-K5 range. Given the compatibility of our reported errors, we do not expect there to be a significant unreported error component in our systemic RV values. We also report the mean of each target's $\sigma_{\text {meas }}$ values and the measurement variance $\left(\sigma_{\text {meas }}^{-2}\right)$ weighted standard deviation of RV measurements for each target as $\sigma_{\text {obs. }}$.

To measure the accuracy of $T_{\text {eff }},[\mathrm{Fe} / \mathrm{H}]$ and $[\alpha / \mathrm{Fe}]$, we compared our measurements for five slowly rotating standard stars spread across our effective temperature range with those in the literature (see table 3 in B16). We saw evidence that our $T_{\text {eff }}$ values are cooler than literature values for both twilight solar and standard star spectra by $25-50 \mathrm{~K},[\mathrm{Fe} / \mathrm{H}]$ is low by -0.03 dex and $[\alpha / \mathrm{Fe}]$ showed evidence of elevation at the 0.01 dex level. Note that these systematics are all within $2 \sigma$. We investigated the accuracy of our $v_{r} \sin (i)$ values by comparing them with those from Terndrup et al. (2002), with which we share 37 of our targets in NGC 2516. Our $v_{r} \sin (i)$ values agreed with a standard deviation of $2.2 \mathrm{~km} \mathrm{~s}^{-1}$. We are unable to resolve values below $\sim 2 \mathrm{~km} \mathrm{~s}^{-1}$, roughly $1 / 3$ of our typical resolution element.
As an additional test of $T_{\text {eff }}$, we compared our values with $T_{\text {eff }}(B-V)$ values computed using the relation of Casagrande et al. (2010) and reddening-corrected colours (Table 1). For cluster members (see Section 5) with $T_{\text {eff }}<6200 \mathrm{~K}$, we measured values about $100 \mathrm{~K}$ cooler than the reddening-corrected colour temperature. Above $6200 \mathrm{~K}$, our values are about $250 \mathrm{~K}$ hotter (Fig. 3). To investigate if this shift in $T_{\text {eff }}$ with $T_{B-V}$ is correlated with the projected rotation, we artificially broadened the stellar lines of the F5V RV standard HIP $31415\left[v_{r} \sin (i) \sim 4.5 \mathrm{~km} \mathrm{~s}^{-1}\right]$ to simulate rotation values between 10 and $50 \mathrm{~km} \mathrm{~s}^{-1}$ and refitted the spectrum. At higher rotation rates, we see an elevation in $T_{\text {eff }}$ and $[\mathrm{Fe} / \mathrm{H}]$ and a decrease in $[\alpha / \mathrm{Fe}]$. For instance, at $40 \mathrm{~km} \mathrm{~s}^{-1}$, we measure an increase in $T_{\text {eff }}$ of $648 \pm 163 \mathrm{~K}$ and $[\mathrm{Fe} / \mathrm{H}]$ of $0.2 \pm 0.08 \mathrm{dex}$ and a decrease of $[\alpha / \mathrm{Fe}]$ by $0.11 \pm 0.08 \mathrm{dex}$. We may see a slight elevation in $T_{\text {eff }}$ at $\sim 10 \mathrm{~km} \mathrm{~s}^{-1}$, though the uncertainty is quite large (Fig. 4). In general, for $v_{r} \sin (i) \lesssim 20 \mathrm{~km} \mathrm{~s}^{-1}$, our stellar properties are largely unaffected.

Since the discrepancy in $T_{\text {eff }}$ is plausibly a side effect of our analysis approach, we computed a model to shift our results on to the widely used scale of Casagrande et al. (2010). We quantified this effect for the combined set of cluster members in NGC 2516 and NGC 2422 by fitting a sigmoid to the difference in $T_{\text {eff }}$ and $T_{B-V}$ (Fig. 3):

$T_{B-V}=T_{\text {eff }}+124 \mathrm{~K}-\frac{415 \mathrm{~K}}{1+e^{-0.0054 \mathrm{~K}^{-1}\left(T_{\text {eff }}-6220 \mathrm{~K}\right)}}$.

One plausible explanation for equation (2) is a combination of an $E(B-V)$ overestimate of $\sim 0.05$ in both clusters and a tendency of our pipeline to overestimate $T_{\text {eff }}$ for more rapidly rotating stars; 66 per cent of stars with a corrected $T_{\text {eff }}>6200 \mathrm{~K}$ have $v_{r} \sin (i)>25 \mathrm{~km} \mathrm{~s}^{-1}$. Non-member stars show a generally linear, albeit offset, agreement with $T_{B-V}$ and are typically $195 \pm 54 \mathrm{~K}$ 

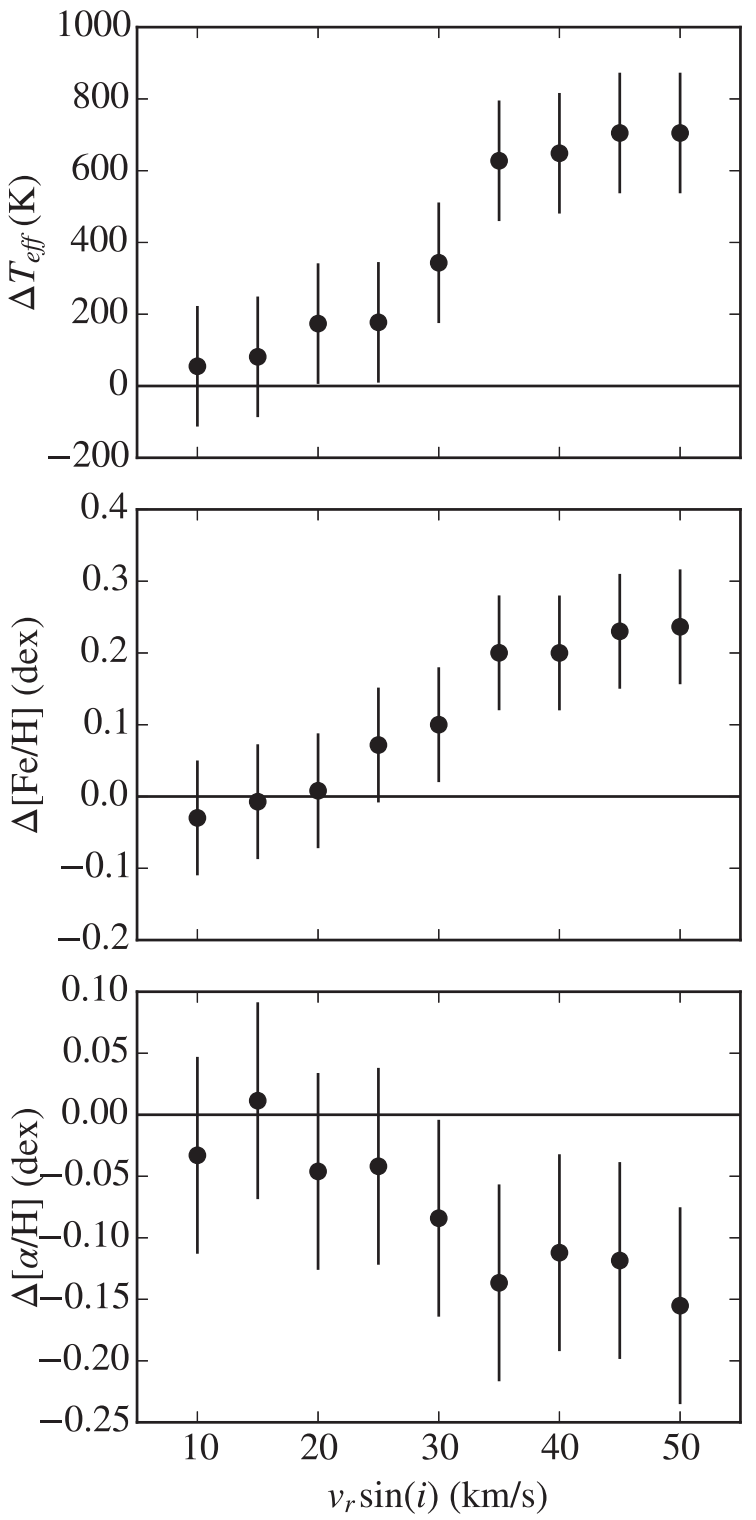

Figure 4. $T_{\text {eff }},[\mathrm{Fe} / \mathrm{H}]$ and $[\alpha / \mathrm{Fe}]$ measurements are affected by artificially broadening the stellar lines in the spectrum of our F5V standard HIP 31415. Error bars show $1 \sigma$ confidence intervals. We stress that we do not see this behaviour in cooler stars.

hotter than their $T_{B-V}$ values despite their on average lower $v_{r} \sin (i)$. We have applied the sigmoid correction to the values we report for members and probable members.

\section{SPECTROSCOPIC BINARIES}

The 9-12 epochs of precise RVs obtained with spacings from $\sim 1 \mathrm{~d}$ to $\sim 1$ yr allow us to identify stars orbited by stellar and near-stellar companions. Because of stellar-activity-induced RV variability (see Section 7), some stars with RV variations above our measurement uncertainty, $\sigma_{\text {meas }}$, may not have an orbiting companion. We, thus, select a variability threshold to help delineate between clear binaries and less obvious cases.

Fig. 5 shows the distribution of $\sigma_{\text {meas }}$ and $\sigma_{\text {obs }}$ for all stars targeted in NGC 2516 and NGC 2422 barring stars mentioned in Section 5.1. The distribution of $\sigma_{\text {meas }}$ shows a Poisson-like peak at $\sim 60 \mathrm{~m} \mathrm{~s}^{-1}$, a tail that extends to $\sim 250 \mathrm{~m} \mathrm{~s}^{-1}$, and then a scattering of stars with

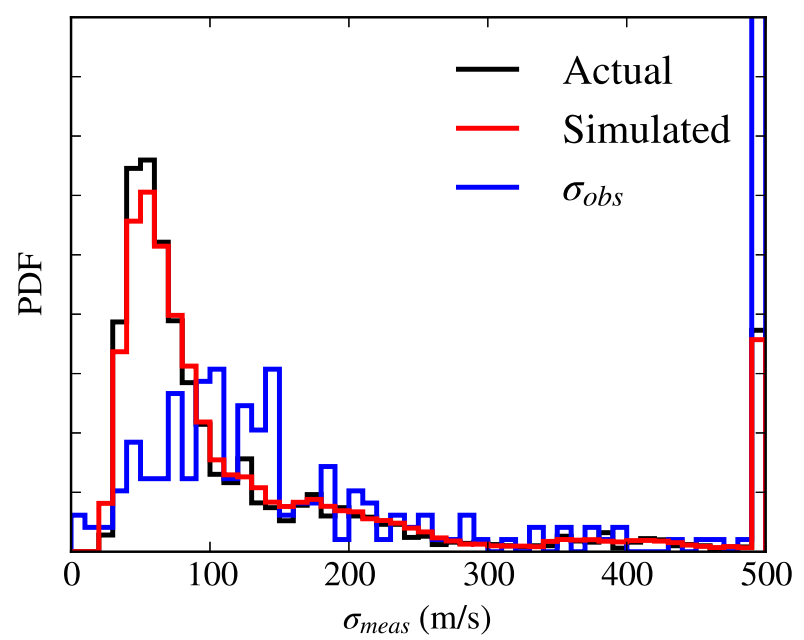

Figure 5. The black line shows the distribution of measurement errors, $\sigma_{\text {meas }}$, for spectra of photometric and candidate members in our sample, exclusive of the seven continuum stars listed in Section 5.1. The red line is the distribution of simulated $\sigma_{\text {meas }}$ values we generate for our companion simulations. The discrepancy to the left of the peak is caused by a higher variance in individual $\sigma_{\text {meas }}$ values for stars with the smallest mean $\sigma_{\text {meas }}$ than our error simulation code captures. The distribution of $\sigma_{\mathrm{obs}}$ is shown for comparison in blue. Its peak in the $500 \mathrm{~m} \mathrm{~s}^{-1}$ bin is $\sim 3.4$ times those of the other two. Values above $500 \mathrm{~m} \mathrm{~s}^{-1}$ have been clipped to that value for this plot. Note that all three histograms have very different normalizations.

larger $\sigma_{\text {meas }}$. The distribution of $\sigma_{\mathrm{obs}}$ shows a broad distribution from 50 to $150 \mathrm{~m} \mathrm{~s}^{-1}$, a tail that roughly matches $\sigma_{\text {meas }}$, and an excess of stars above $500 \mathrm{~m} \mathrm{~s}^{-1}$, which is $\sim 3.4$ times that expected from measurement errors alone.

Under the assumption that most stars have constant RVs, the difference between the peaks of the $\sigma_{\text {meas }}$ and $\sigma_{\text {obs }}$ distributions can serve as a proxy for the characteristic amplitudes of any stellaractivity-induced RV variability. Based on this comparison, we adopt a stellar variability

$\sigma_{\text {stel }} \equiv \sqrt{\sigma_{\text {obs }}^{2}-\overline{\sigma_{\text {meas }}^{2}}}$

where $\sigma_{\text {stel }}=300 \mathrm{~m} \mathrm{~s}^{-1}$ is the dividing line between what we will refer to as spectroscopic single and spectroscopic binary stars in our sample. We emphasize that, as defined, these spectroscopic binary stars could have brown dwarf $\left(M \leq 0.07 \mathrm{M}_{\odot}\right)$ or planetary $(M \leq$ $13 \mathrm{M}_{\mathrm{Jup}}$ ) companions. We also require that the RV variability is statistically significant (Section 8) to account for the small number of stars with large measurement errors.

We identified 40 spectroscopic binaries in our sample of 126 stars in the field of NGC 2516 and 22 in our sample of 125 stars in the field of NGC 2422. We find a median $\sigma_{\text {obs }}$ of $3200 \mathrm{~m} \mathrm{~s}^{-1}$, with values ranging from 310 to $38000 \mathrm{~m} \mathrm{~s}^{-1}$. Eight each have $\sigma_{\mathrm{obs}}=300-500 \mathrm{~m} \mathrm{~s}^{-1}$ and $\sigma_{\mathrm{obs}}=500-1000 \mathrm{~m} \mathrm{~s}^{-1}$ with the remainder being above $1000 \mathrm{~m} \mathrm{~s}^{-1}$. Eight of our spectroscopic binaries (four in each cluster field) are clear double-lined binaries. The parameters we report are for the stronger of the pair, but errors for these stars should be treated with a degree of caution. We exclude five stars with $\sigma_{\text {stel }}>300 \mathrm{~m} \mathrm{~s}^{-1}$ that have $\sigma_{\text {meas }}>600 \mathrm{~m} \mathrm{~s}^{-1}$ and $v_{r} \sin (i)>35 \mathrm{~km} \mathrm{~s}^{-1}$ as their RV variations were not statistically significant $\left(P_{\mathrm{v}} \leq 0.96\right.$; Section 8$)$. We use the codes B or SB2 to denote spectroscopic or double-lined spectroscopic binaries, respectively, in Tables A1 and A2. We are able to recover candidate periods for a number of these binaries in Section 8.1. RV curves for these stars are provided in Appendix B (online only). 


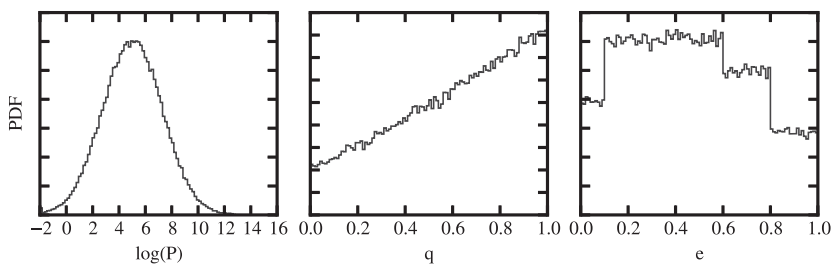

Figure 6. The period, mass ratio and eccentricity distributions of stellar binaries in our companion simulations. Though not evident in the plot, the mass ratio and eccentricity distributions are a function of the period. Distributions are based on those given in DK13.
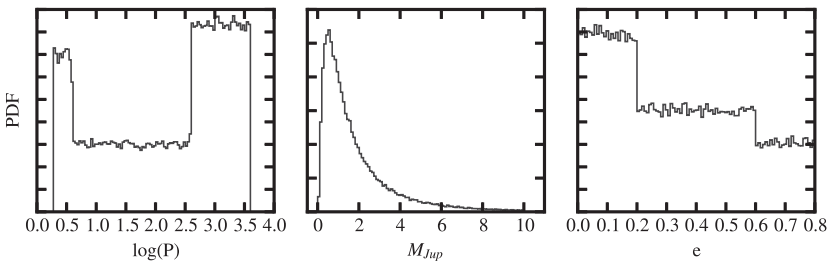

Figure 7. The period, mass ratio and eccentricity distributions of exoplanetary companions in our companion simulations. Distributions are based on those given in Udry \& Santos (2007).

\subsection{Companion simulations}

The temporal sampling and precision of our survey prevented us from detecting long-period variables and the lowest mass companions. To investigate these factors and their effects upon both the binary criterion described above and the results presented in later sections, we performed Monte Carlo simulations of binary stellar and exoplanetary systems. Here, we describe the technical aspects of our simulations; later sections use these simulations to consider the RV variability we would expect, to quantify companion detectability and to investigate how likely binaries are to pass our membership tests. Figs 6 and 7 show the input distributions for period, eccentricity and companion mass for the binary and exoplanet populations. These are according to Duchêne \& Kraus (2013, hereafter DK13; for binaries) and Udry \& Santos (2007, for exoplanets). We chose a total exoplanet fraction such that we would expect 1.2 per cent of stars to have hot Jupiter companions consistent with Wright et al. (2012), and we start with a binary fraction of 45 per cent consistent with DK13. We adjust this value when investigating the binary fraction.

We simulated a sample of 150000 systems around a $1 \mathrm{M}_{\odot}$ star with carefully generated measurement errors for each simulated RV to match the properties of our data set. These errors were generated by creating a sample of fake mean $\sigma_{\text {meas }}$ values from the distribution of $\sigma_{\text {meas }}$ values in our data. We then sampled a Gaussian distribution with width corresponding to the mean spread in $\sigma_{\text {meas }}$ for each target $\left(\sim 10 \mathrm{~m} \mathrm{~s}^{-1}\right)$ to perturb the fake errors chosen for each star. In this way, we generated unique errors on each simulated RV measurement that mirror our sample (see the red line in Fig. 5). We observe each simulated star at the sample cadence of each cluster by sampling Gaussians located at each RV where each Gaussian is given the width of the corresponding simulated error and computed $\sigma_{\text {obs }}\left(\right.$ Section 3.2) and $P_{v}$ (Section 8) for each simulated target. When investigating how binaries fare in our membership test, we included random systemic velocities for each star using our observed cluster velocity dispersions of $734 \mathrm{~m} \mathrm{~s}^{-1}$ in NGC 2516 and $750 \mathrm{~m} \mathrm{~s}^{-1}$ in NGC 2422 (Section 6).

We find our $300 \mathrm{~m} \mathrm{~s}^{-1}$ binarity threshold will identify 69 per cent of the stellar binaries in our sample as single members, though
95 per cent these have periods longer than $25 \mathrm{yr}$. Similarly, we find the threshold imposes a 9 percent false-positive rate (with stated binary and exoplanet fractions, which are lower with increasing binary fraction), $1 / 5$ of which (1.8 percent of the total) would be (very massive) planetary or brown dwarf companions flagged as binaries.

\section{MEMBERSHIP}

Because stars in an open cluster are expected to have formed at the same time and from the same parent cloud, and to still be moving through space together, numerous age, compositional and kinematic diagnostics can be used to identify cluster members. While multiple diagnostics will, in general, improve the overall accuracy of membership lists, their use can also lead to the exclusion of stars in peculiar evolutionary or dynamical stages (e.g. interacting star-star or star-planet systems). Since RVs were not used in identifying cluster members from J01 or P03, the precise RVs achieved in this study provide a very valuable check on membership.

We determine membership probabilities by assuming that the RVs of the stars in our sample are drawn either from a Gaussian $\mathrm{RV}$ distribution centred on the cluster (for members) or from a Besançon distribution (Robin et al. 2003) of Galactic stars along the cluster line of sight (for non-members). The Besançon distribution is a Galactic stellar-population synthesis model that provides broad agreement with surveys and includes kinematics. To compute the RV membership probability, $P_{\mathrm{RV}}(v)$, we first computed an observed probability density function (PDF) from the normalized sum of Gaussian PDFs for each of our target stars. That is, we locate a Gaussian at each measured RV with $\sigma$ corresponding to the bootstrapped errors on the weighted mean (B16). We then fitted the PDF with a weighted sum of a Gaussian and the Besançon PDF and computed a membership probability for each star using

$P_{\mathrm{RV}}(v)=\frac{f_{\text {cluster }}(v)}{f_{\text {cluster }}(v)+f_{\mathrm{MW}}(v+c)}$,

where $f_{\text {cluster }}(v)$ is the fitted Gaussian PDF component and $f_{\mathrm{MW}}(v+c)$ is the Milky Way component with a constant to allow for small shifts in the centre of the distribution. We adopt targets with $P_{\mathrm{RV}}>50$ percent as RV members such that the balance of probability is for membership. This threshold corresponds to $\sim 2.5 \sigma_{R V}$ in both clusters. Figs 8 and 9 show histograms of our target RVs and the Besançon RVs, our PDF and the best-fitting model, and a comparison of the resulting cumulative distribution functions. Considering the overlap of the best-fitting Besançon and Gaussian RV distributions for each cluster, we expect this to yield a false-positive rate of 12 per cent in NGC 2516 and 13 per cent in NGC 2422. This fitting process also yields values for each cluster's observed velocity dispersion (Gaussian sigma), which we discuss in Section 6.

Our Monte Carlo simulations show this membership test is strongly biased against large-amplitude RV variables. At our observing cadence - for either cluster - 30 per cent of the time we would observe a simulated binary system to have $P_{\mathrm{RV}}<50$ per cent. Large-amplitude binaries, e.g. those we identify as spectroscopic binaries, fare much worse: $\sim 67$ per cent of simulated systems we flag as an RV binary will fail a $P_{\mathrm{RV}}=50$ per cent cut. To adopt a more forgiving approach, we consider stars with $\sigma_{\text {stel }}>300 \mathrm{~m} \mathrm{~s}^{-1}$ that have RV within $2 \sigma_{\overline{R V}}$ of the $P_{\mathrm{RV}}=50$ per cent threshold to be probable members. This relaxed criterion reduces the exclusion of large-amplitude binaries from 67 percent to 31 per cent in our simulation. 

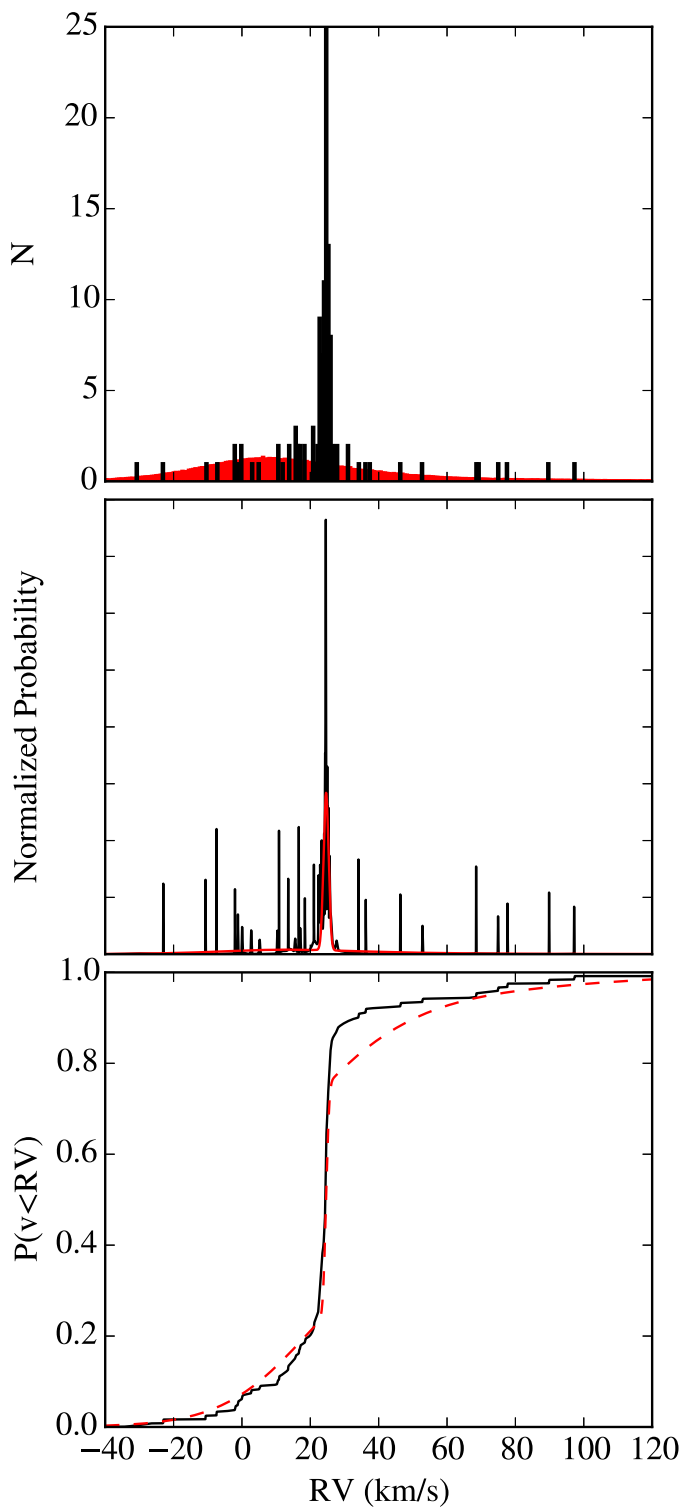

Figure 8. Top: Histogram of the measured RVs of our targets in NGC 2516 superimposed over a scaled histogram of stars in a Besançon model along the line of sight to NGC 2516 with matching cuts on $V$ and $B-V$. Middle: PDF constructed from our RVs and their measurement errors. Our best-fitting model is drawn as a solid red line. Bottom: The continuous distribution functions for our PDF (solid) and the best-fitting model (dashed). The discrepancy to the right of the cluster is a result of fewer contaminants than predicted by the Besançon model.

The results of the membership analysis are summarized in the following sections. A small number of stars could not be tested for membership using the procedure outlined or are otherwise notable despite their RV non-member status and are described in Section 5.1. Membership and the short-period multiplicity of stars in NGC 2516 and NGC 2422 are discussed in Sections 5.2 and 5.3, and a candidate association, perhaps related to the Monoceros ring, in Section 5.4. The membership probabilities, $P_{\mathrm{RV}}$, are listed in Tables A1 and A2. We use the codes $\mathrm{N}$ (non-member), $\mathrm{P}$ (probable member) and $\mathrm{M}$ (member) to denote the levels of RV membership certainty. Two stars described in the following section are given the code $\mathrm{X}$, as no determination was possible.

\subsection{Continuum stars and probable contaminants}

Twenty-seven stars exhibit a diffuse interstellar band (DIB) at $7224.2 \AA$ (Fig. 10 and Herbig \& Soderblom 1982). None of the 22 for which we are able to obtain RVs pass our RV membership test, suggesting that there is insufficient column depth within a few hundred parsecs to produce a notable feature. This suggests that the presence of the DIB indicates a non-member target. The diffuse band does not pose any difficulty to our fits, nor does it perturb our results, as verified by masking out the region. We do find that stars with the feature generally yield lower $\log (g)$ values relative to cluster members if we allow $\log (g)$ to vary, further suggesting they are distant giants.

Five stars exhibiting the DIB and with colours corresponding to mid-F and late-G/early-K spectral types have either few, exceptionally broad or no discernible lines (Fig. 10) and caused our fitting pipeline to fail. A visual comparison with the most rapid rotators that we are able to fit $\left(\sim 90 \mathrm{~km} \mathrm{~s}^{-1}\right)$ suggests two of these (147012316 and 147-012471) would require $v_{r} \sin (i) \sim 120 \mathrm{~km} \mathrm{~s}^{-1}$ for an approximate match with the template spectrum. The remaining three show no evidence of any photospheric absorption features, even when compared to templates with $v_{r} \sin (i) \sim 200 \mathrm{~km} \mathrm{~s}^{-1}$. Tables A1 and A2 give the parameters for these stars as missing data with type $\mathrm{C}$ for continuum, as we are not able to fit their spectra and we flag them as non-members due to the DIB. Note that these could be heavily extincted, background early-type stars that would not have appreciable lines in this region independent of rotation, which would also be expected. One star was observed using a fibre with very poor throughput and never attained sufficient $\mathrm{S} / \mathrm{N}$ for analysis. It is noted with an X for both type and membership. We now describe some specific properties of the targets that have exceptionally weak or scarcely discernible lines.

146-012353 is listed by $\mathrm{J} 01$ as photometric single member 6337 in NGC 2516. It has $T_{B-V}=6900 \mathrm{~K}$. It is 0.08 arcsec from a source given in Damiani et al. (2003, hereafter D03) as having $\log \left(L_{\mathrm{X}}\right)<29.75 \mathrm{erg} \mathrm{s}^{-1}$ based on Chandra observations. Visual inspection suggests $v_{r} \sin (i)$ in excess of $200 \mathrm{~km} \mathrm{~s}^{-1}$.

147-012316 is listed by J01 as photometric single member 8920 in NGC 2516. It has $T_{B-V}=6840 \mathrm{~K}$. D03 reports it as having a flux of $1.36 \pm 0.4810^{-6}$ counts s${ }^{-1} \mathrm{~cm}^{-2}$ and $\log \left(L_{X}\right)$ of $28.85 \mathrm{erg}$ $\mathrm{s}^{-1}$. Visual inspection suggests $v_{r} \sin (i)$ is between 120 and $150 \mathrm{~km} \mathrm{~s}^{-1}$.

147-012471 is listed by $\mathrm{J} 01$ as photometric single member 12302 in NGC 2516. It has $T_{B-V}=6625 \mathrm{~K}$. It is 0.134 arcsec from a source in D03 with $\log \left(L_{\mathrm{X}}\right)<29.96 \mathrm{erg} \mathrm{s}^{-1}$ and $0.282 \mathrm{arcsec}$ from source 272 of Pillitteri et al. (2006), which is reported to have a

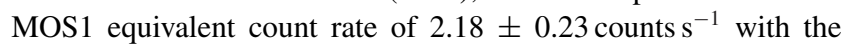
$X M M-N e w t o n$ EPIC camera. Visual inspection suggests $v_{r} \sin (i)$ of $\sim 120 \mathrm{~km} \mathrm{~s}^{-1}$.

378-036424 is listed as member 956 in NGC 2422 by P03 and has $T_{B-V}=5650 \mathrm{~K}$. Visual inspection suggests $v_{r} \sin (i)$ in excess of $200 \mathrm{~km} \mathrm{~s}^{-1}$.

379-036213 is one of our 25 UCAC4 targets and has $T_{B-V}=5330 \mathrm{~K}$. Visual inspection suggests $v_{r} \sin (i)$ in excess of $200 \mathrm{~km} \mathrm{~s}^{-1}$.

We also note one metal-poor high-velocity star in the NGC 2516 sample. 146-012596 has an RV of $335.901 \pm 0.048 \mathrm{~km} \mathrm{~s}^{-1}$ and does not show any sign of RV variability. We find a rotation rate of $3.1 \mathrm{~km} \mathrm{~s}^{-1}$ and note that its iron abundance runs into the lower edge of our grid, suggesting the true value may be less than -1 dex. It exhibits the DIB at $7224 \AA$. 

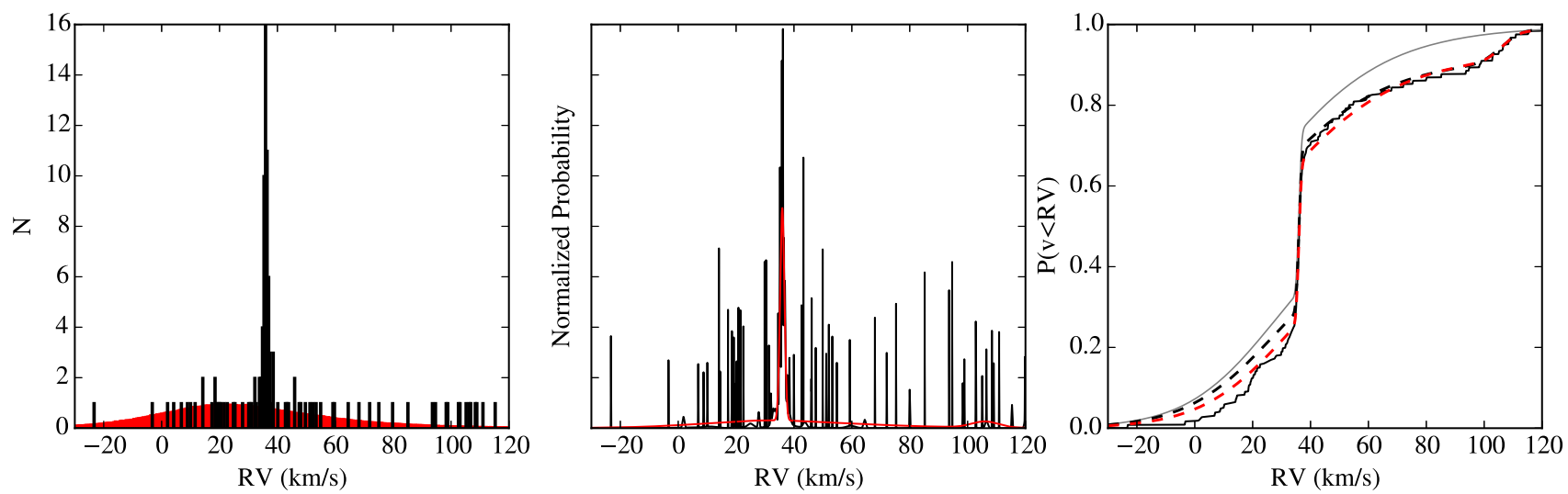

Figure 9. Left: Histogram of the measured RVs of our targets in NGC 2422 superimposed over a scaled histogram of stars in a Besançon model along our pointing's line of sight with matching cuts on $V$ and $B-V$. Middle: PDF constructed from our RVs and their measurement errors. Our adopted model is plotted as a red line. Right: The continuous distribution functions for our data (solid) and the best-fitting models. The thin grey line is for a simple Besançon + Gaussian model. The dashed black line includes a second Gaussian for the overdensity of stars at $\sim 106 \mathrm{~km} \mathrm{~s}^{-1}$ and the dashed red line includes both the second Gaussian and an allowance for a mean shift (measured to be $-4.9 \mathrm{~km} \mathrm{~s}^{-1}$ ) in the Besançon RVs. The modifications alter $P_{\mathrm{RV}}$ by no more than 1.2 per cent with a mean of 0.2 per cent and do not affect the classifications of any of our stars.

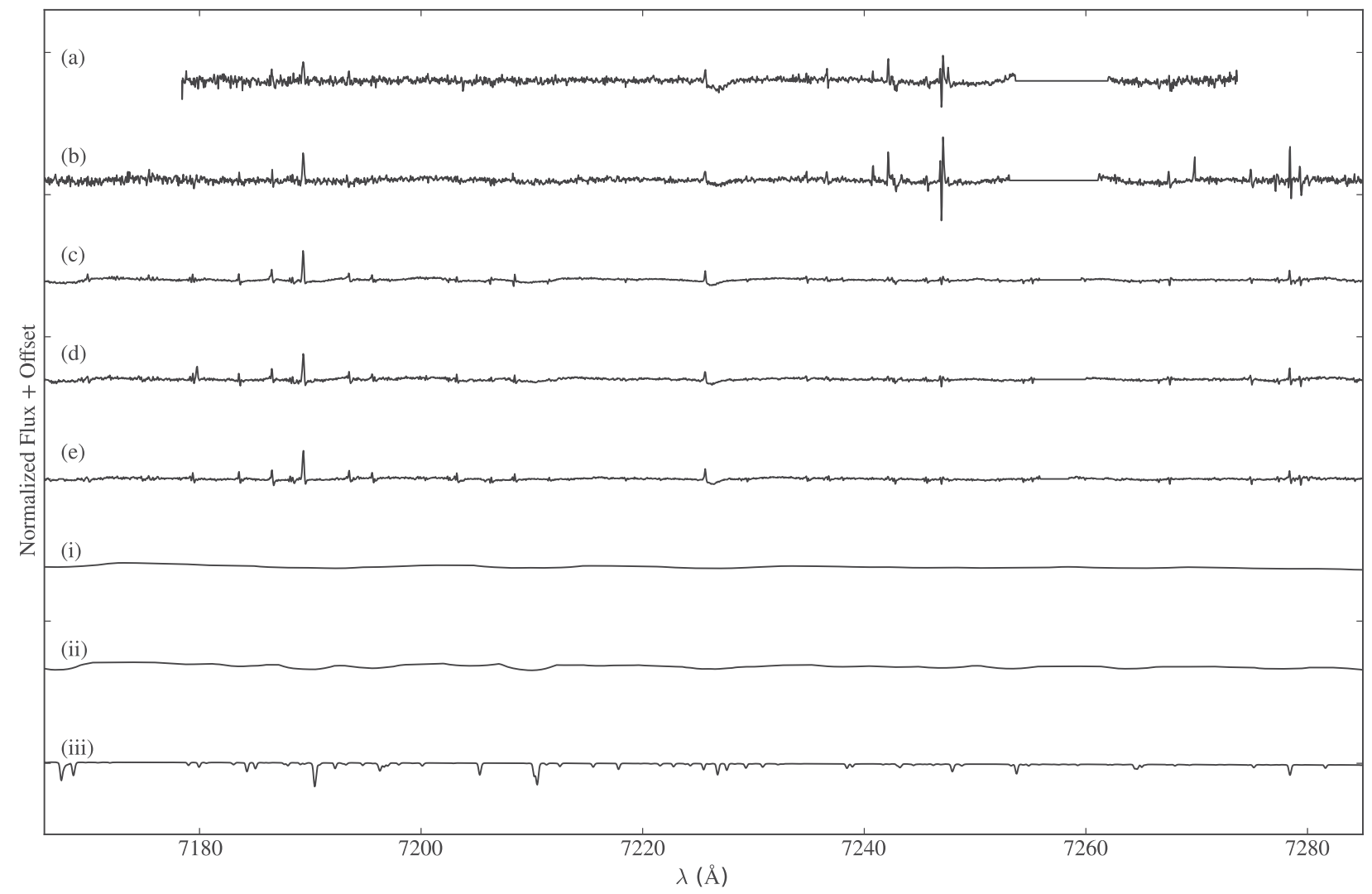

Figure 10. Spectra (a-e) of the five near featureless objects in our sample. The individual epochs have been (mostly) cleaned of telluric absorption and emission lines and summed to yield these high S/N spectra. The spectrum of 379-036213 (a) is clipped at either end as the data in some of the component epochs fall below our minimum S/N limit for fitting. A DIB is visible in the spectra at $7224 \AA$. The spectra are as follows: (a) $379-036213: V=14.2, B-V=0.87, \mathrm{~S} / \mathrm{N}=$ 150. (b) 378-036424: $V=15.0, B-V=0.75, \mathrm{~S} / \mathrm{N}=79$. (c) $147-012471: V=12.0, B-V=0.53, \mathrm{~S} / \mathrm{N}=364$. (d) $147-012316: V=11.7, B-V=0.47, \mathrm{~S} / \mathrm{N}$ =336. (e) 146-012353: $V=11.7, B-V=0.46, \mathrm{~S} / \mathrm{N}=343$. Visually, 147-012471 and 147-012316 (c and d) have faint features, suggesting they are rotating

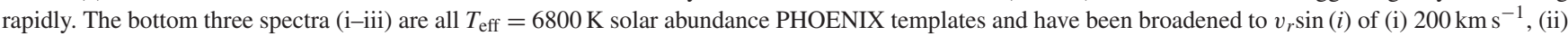
$100 \mathrm{~km} \mathrm{~s}^{-1}$ and (iii) $20 \mathrm{~km} \mathrm{~s}^{-1}$ for comparison.

Finally, 378-036788 is identified by P03 as an early-type member in NGC 2422. This star entered our sample via a cross-matching error and has no discernible features. We list it as type $\mathrm{C}$ in Table A2 with a membership flag of $\mathrm{X}$ for no data.

\subsection{Membership and short-period multiplicity in NGC 2516}

Of the 126 photometric members targeted in NGC 2516, we identified 81 stars as RV members. Of these 81 , we classify 54 as RV 
single and 27 as RV binary. Two RV members are labelled photometric non-members by J01 (146-012470 and 147-012335, each failing one of J01's three tests). 12 of the RV members are classified by $\mathrm{J} 01$ as photometric binaries. Ten of the $51 \mathrm{~J} 01$ single members fail our RV membership cut. J01 estimated contamination fractions of $15 \pm 8$ per cent for single and $30 \pm 11$ per cent for binary members in their membership list over the relevant colour range and thus, we could reasonably expect to find RV non-members at this level. Our simulation predicts 11 per cent of true cluster members will be excluded by our membership test assuming cluster members have (systemic) Gaussian RVs distributed according to the measured cluster velocity dispersion $\left(734 \mathrm{~m} \mathrm{~s}^{-1}\right.$; Section 6). 37 per cent of the excluded members are predicted to be binaries with 99 per cent (95 per cent) having $P>8.6(25)$ yr.

Eight of the RV binary members were included by our relaxed membership criterion and 18 were identified as single members in J01. We find 29 of the photometric binaries in J01 fail our RV binarity test and 17 fail our RV membership cut. After correcting for our sensitivity, our findings agree with their contamination estimates for single members but find they underestimate the photometric binary contamination by $\sim 22$ percent, assuming our RV membership is correct.

Using the RV binary criteria described in Section 4, we find an observed RV binary fraction of $33 \pm 5$ percent. Our sample is subject to a false-positive rate of 9 percent on RV binarity and false positive and false-negative rates of 12 percent and 31 percent, respectively, on RV membership. This suggests that four of the non-member RV binaries are RV members, two of the 27 RV binary members are single members, and three of the 27 are binary but not members. With these corrections, we would predict $25[\approx 0.91 \times(27+0.31 \times 13-0.12 \times 27)]$ $\mathrm{RV}$ binaries with $\sigma_{\text {stel }}>300 \mathrm{~m} \mathrm{~s}^{-1}$ in a sample of 78 $[\approx 81-0.12 \times 81+0.31 \times 13+0.05 \times 28] \mathrm{RV}$ members for an observed RV binary fraction of $32 \pm 6$ per cent.

Our companion simulation, presented in Section 4.1, predicts only 15 percent of stars should be flagged as RV binary $\left(\sigma_{\text {stel }}>300 \mathrm{~m} \mathrm{~s}^{-1}\right)$ under the assumption that the overall binary fraction is 45 per cent, matching the field population. We would expect 27 per cent flagged at an overall binary fraction of 85 per cent as reported in J01. A 100 percent binary fraction would result in 31 per cent of stars being flagged as RV binary, consistent with what is measured here. We caution that this assumes the same period distribution as that in the field. For comparison, in J01, 47 of our 126 targets (37 per cent) were flagged as photometric binaries, though recall we do not flag 12 of their photometric binaries as RV binaries. We investigate the potential composition of these binaries in Section 8.

Extensive work in the field shows typical multiplicity fractions of $62 \pm 3$ per cent for solar-mass stars $\left(0.7-1.3 \mathrm{M}_{\odot}\right.$; see the review in DK13) and work on open clusters has shown a similar picture with a value of about 65 per cent (DK13). Our result is consistent with J01's finding of $85 \pm 15$ per cent, especially considering that mass segregation may be influencing our finding, as all of our targets are near the cluster centre. Indeed, a perusal of cluster colourmagnitude diagrams in, for example, the WEBDA data base will quickly reveal how striking the binary sequence is in NGC 2516.

Of the $41 \mathrm{RV}$ non-members, 13 are identified as spectroscopic binaries, one of which is a double-lined binary. Six of our RV spectroscopic binary non-members were listed by J01 as photometric single members and eight as photometric binary members. We are unable to assess fully the membership status of the three featureless stars in NGC 2516 (Section 5.1). Although J01 classified them as single members, we classify them as RV non-members due to the DIB.

\subsection{Membership and short-period multiplicity in NGC 2422}

Of the 100 photometric members and 25 candidate members targeted in NGC 2422, we identified 57 stars as RV members, 11 of which are RV binary. Of the 25 candidate members, we find seven to be RV single members and five to be RV binary members. P03 does not attempt to identify binaries using their photometry, so we are unable to compare our results, as in NGC 2516. Five RV binaries were included by our relaxed RV binary membership criterion. Our simulation predicts 14 per cent of true cluster members will be excluded by our membership test, assuming cluster members have (systemic) Gaussian RVs distributed according to the measured cluster velocity dispersion (Section 6). 37 per cent of the excluded members are predicted to be binaries with 99 per cent ( 95 per cent) having $P>8(23) \mathrm{yr}$.

Note seven stars - 378-036692, 378-036906, 379-035967, 379035982, 377-035049, 378-036136 and 378-036960 - pass our membership test but are somewhat removed from the rest of the main sequence (MS) in the temperature-magnitude space (Fig. 11): three are below and four above. The relative areas of the Besançon and cluster Gaussian PDFs suggest that we could expect seven field stars to pass as RV members. All but 378-036960 yield $\log (g)$ values broadly consistent with MS stars if we allow $\log (g)$ to vary and we also note 378-036960 is one of only two mid-F stars that pass our membership test and have $v_{r} \sin (i) \lesssim 25 \mathrm{~km} \mathrm{~s}^{-1}$. Two of the seven, 378-036692 and 379-035967, were flagged as members by P03. The rest were targets selected from UCAC4. Three of them - 378-036906, 378-036136, 378-036960 - have errors of $B-V$ in excess of $0.13 \mathrm{mag}$. We flag these three stars as probable members instead of members.

Based on the RV binary criteria described in Section 4, we find an observed RV binary fraction of $19 \pm 5$ percent. With a falsepositive rate of 9 percent on RV binarity and false-positive and false-negative rates of 13 per cent and 31 per cent, respectively, on $\mathrm{RV}$ membership, we expect that three of the 11 non-member RV binaries are, in fact, RV binary members, one of the 11 member RV binaries is not binary, and one of the $11 \mathrm{RV}$ members is not a member. With these corrections, we would predict $12 \mathrm{RV}$ binaries with $\sigma_{\text {stel }}>300 \mathrm{~m} \mathrm{~s}^{-1}$ in a sample of $59 \mathrm{RV}$ members for an observed RV binary fraction of $20 \pm 5$ per cent. Our simulation predicts a cluster binary fraction of $62 \pm 16$ per cent would produce our observed RV binary fraction, perfectly consistent with DK13. We investigate the potential composition of these binaries in Section 8.

Of the $68 \mathrm{RV}$ non-members, 11 are RV binaries, two of which are double-lined binaries. We are unable to assess fully the status of the two previously mentioned featureless stars (one from P03 and one from UCAC4) in NGC 2422. We classify them as RV non-members due to the DIB.

\subsection{A distant association}

In NGC 2422, we noted an overdensity of 11 stars with a mean RV of $\sim 107 \mathrm{~km} \mathrm{~s}^{-1}$, well removed from the cluster and the Besançon model distribution. We introduced an additional Gaussian component to our membership model that accounted for this grouping and substantially improved our continuous distribution function (Fig. 9). Note that the second Gaussian does not appreciably affect our membership probabilities in NGC 2422. These stars cluster at $106.8 \pm 1.3 \mathrm{~km} \mathrm{~s}^{-1}$ with $\sigma_{\mathrm{RV}}$ of $3.76 \mathrm{~km} \mathrm{~s}^{-1}$. They have a mean 

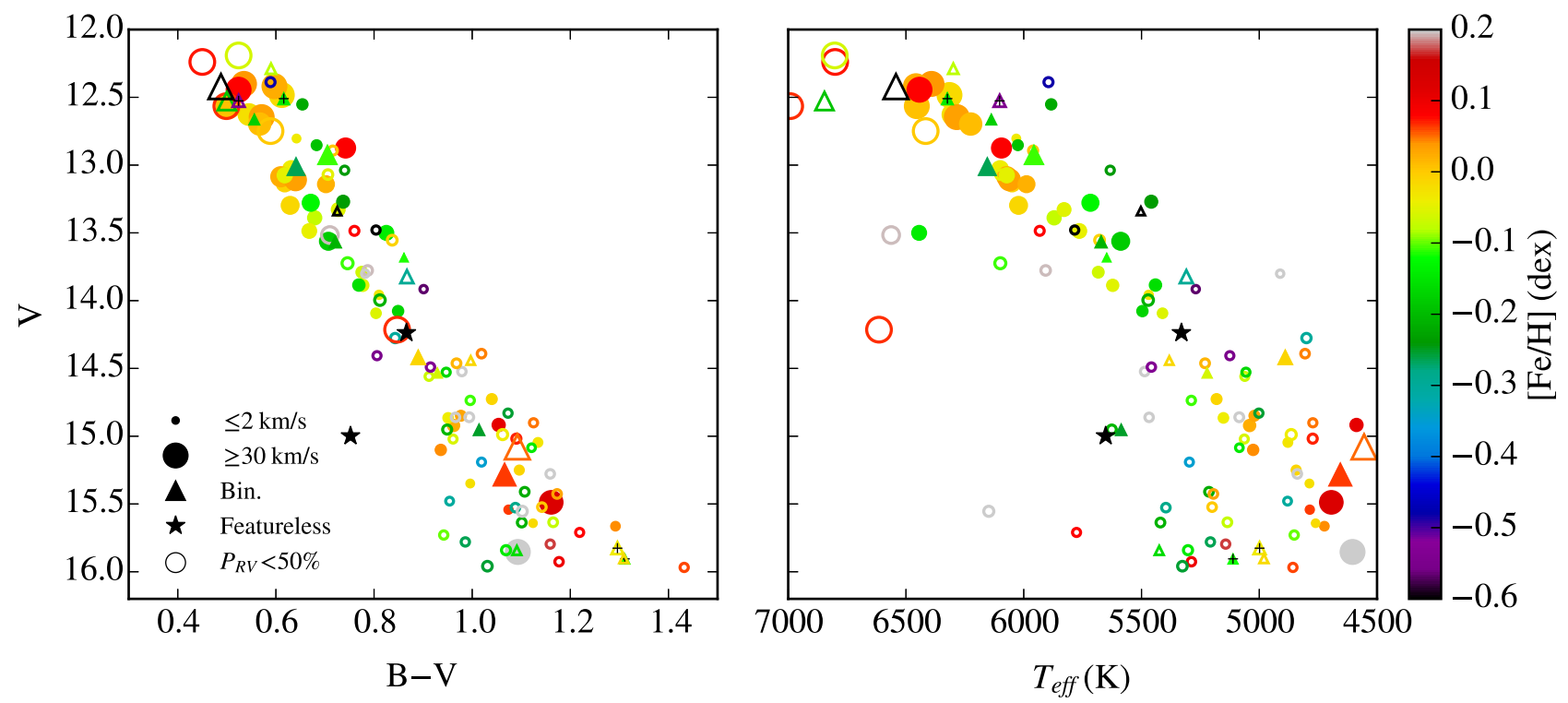

Figure 11. Colour-magnitude (left) and temperature-magnitude (right) diagrams for targets in NGC 2422. Stars we identified as RV members (Section 5) are shown as filled circles or, for RV binaries, triangles. Double-lined binaries have a tiny black plus sign on them. The colour indicates [Fe/H] and the point size $v_{r} \sin (i)$. Unfilled points show the same for targets that do not pass our RV membership test. The filled black stars correspond to the featureless spectra

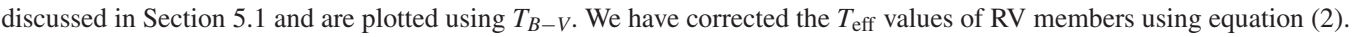

Table 5. Cluster properties.

\begin{tabular}{lcccccc}
\hline Cluster & $N$ & $\begin{array}{c}\mathrm{RV} \\
\left(\mathrm{km} \mathrm{s}^{-1}\right)\end{array}$ & $\begin{array}{c}\sigma_{\mathrm{RV}} \\
\left(\mathrm{m} \mathrm{s}^{-1}\right)\end{array}$ & $\begin{array}{c}{[\mathrm{Fe} / \mathrm{H}]} \\
(\mathrm{dex})\end{array}$ & $\begin{array}{c}{[\alpha / \mathrm{Fe}]} \\
(\mathrm{dex})\end{array}$ & $\begin{array}{c}\text { Binary fraction } \\
(\text { percentage })\end{array}$ \\
\hline NGC 2516 & 81 & $24.50 \pm 0.12$ & $734 \pm 104$ & $-0.08 \pm 0.01$ & $0.03 \pm 0.01$ & $100_{-15}^{+0}$ \\
NGC 2422 & 57 & $35.97 \pm 0.09$ & $750 \pm 65$ & $-0.05 \pm 0.02$ & $0.02 \pm 0.01$ & $62 \pm 16$ \\
\hline
\end{tabular}

$v_{r} \sin (i)$ of $3.9 \pm 0.7 \mathrm{~km} \mathrm{~s}^{-1}$ and all exhibit a DIB at $7224.2 \AA$. When fitting allowed $\log (g)$ to vary, we found values $\sim 2.25 \mathrm{dex}$ lower than cluster members in NGC 2422. As a second test, we fitted them using the pipeline of Walker, Olszewski \& Mateo (2015), which indicated $\log (g)$ of $0.5-1.5$. These tests strongly suggest the stars are giants or supergiants, though there is no star formation at the required distance for a supergiant. Assuming 1.22 mag of extinction and $0.39 \mathrm{mag}$ of reddening as per Schlafly \& Finkbeiner (2011), an average $V$ magnitude of 15.5 , and a dereddened colour temperature of $\sim 5500 \mathrm{~K}$, we would suggest that these stars are either G2 supergiants at $150 \mathrm{kpc}$ or G5 giants at $\sim 13 \mathrm{kpc}$. NGC 2422 is located at $l=231, b=+3$ and it is possible that these stars may be part of some stream or ring along the line of sight. Note that the Monoceros ring is at higher Galactic latitudes (Slater et al. 2014) and it is, thus, possible that this represents a detection of that material at a lower latitude where detection with photometry is impractical due to confusion with the disc.

\section{CLUSTER RVS, METALLICITY AND PROJECTED ROTATION}

We now turn our attention to the aggregate properties of our targeted clusters: systemic RV, observed velocity dispersion, iron and $\alpha$ element abundances, and binary fractions.

The systemic RV for each cluster was computed in the same manner as that for each target (Section 3.2), excluding both RV non-members and the small number of RV members included by our relaxed RV membership criterion. The cluster velocity dispersions are the Gaussian standard deviation taken from our fit of equation (3) as described in Section 5. These values are reported in Table 5. Our RV for NGC 2516 agrees with the result of Terndrup et al. (2002) but the authors do not report a velocity dispersion for the cluster. We are the first authors to report detailed RV data for NGC 2422.

$[\mathrm{Fe} / \mathrm{H}]$ and $[\alpha / \mathrm{Fe}]$ values were derived using a Gaussian kernel density estimation on the member stars (Figs 12 and 13). Values are reported in Table 5. In NGC 2516, our iron abundance of $-0.08 \pm 0.01 \mathrm{dex}$ is only slightly inconsistent with the value of $0.01 \pm 0.07$ dex reported by Terndrup et al. (2002) using the spectroscopy of two stars, neither of which is in our sample. We measure an $\alpha$-element enhancement of $0.03 \pm 0.01 \mathrm{dex}$, though we acknowledge this value may be driven down by our optimizer's interaction with the high rotation rates in hotter stars (Fig. 4). In NGC 2422, our iron abundance of $-0.05 \pm 0.02 \mathrm{dex}$ is moderately inconsistent with the $+0.11 \pm 0.1$ dex value reported by Nissen (1988) using Strömgren photometry of 11 stars. We measure an $\alpha$-element enhancement of $0.02 \pm 0.01$ dex.

We separated both $[\mathrm{Fe} / \mathrm{H}]$ and $[\alpha / \mathrm{Fe}]$ into spectral-type groups to investigate any trends with temperature, as is illustrated in Figs 12 and 13. In both clusters, we measured lower iron abundances for $\mathrm{G}$ stars than the cluster as a whole $(-0.04 \pm 0.022$ dex for NGC 2516 and $-0.04 \pm 0.028 \mathrm{dex}$ for NGC 2422) and also note elevated abundances in F stars, especially in NGC $2516(+0.07 \pm 0.022$ dex $)$. This latter point is expected given the higher stellar rotation and its effect on our $[\mathrm{Fe} / \mathrm{H}]$ values (Section 3.2 and Fig. 4). $\alpha$-element abundances present a more complicated picture with some indication of bimodality in all spectral-type bins. We posit this indicates our data may benefit from a traditional abundance analysis to test 

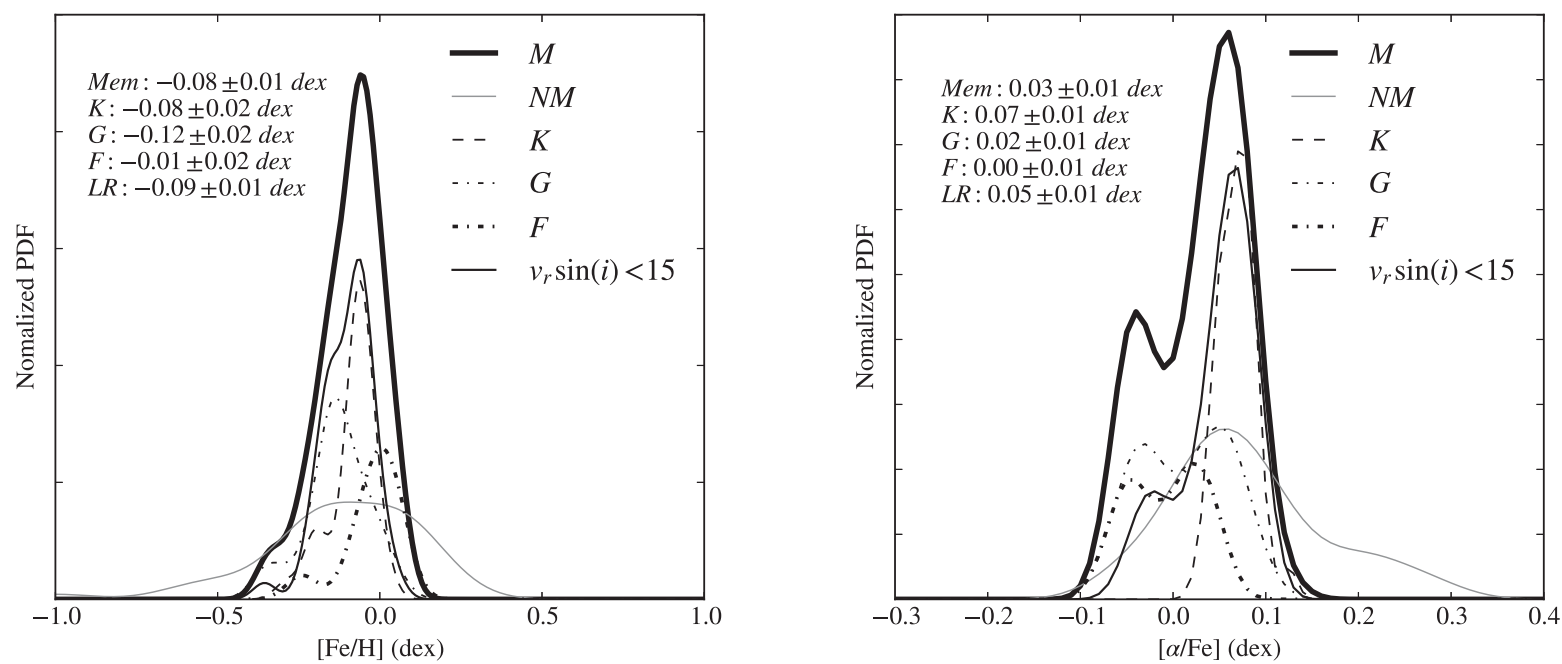

Figure 12. Gaussian kernel density estimates of $[\mathrm{Fe} / \mathrm{H}]$ (left) and $[\alpha / \mathrm{Fe}]$ (right) in NGC 2516. The thick black line shows the full RV member population and the thin grey line the RV non-member sample. The other lines show the distributions for various subsets of our RV member sample. LR stands for low-rotation and gives the mean for the $v_{r} \sin (i)<15 \mathrm{~km} \mathrm{~s}^{-1} \mathrm{RV}$ member sample.
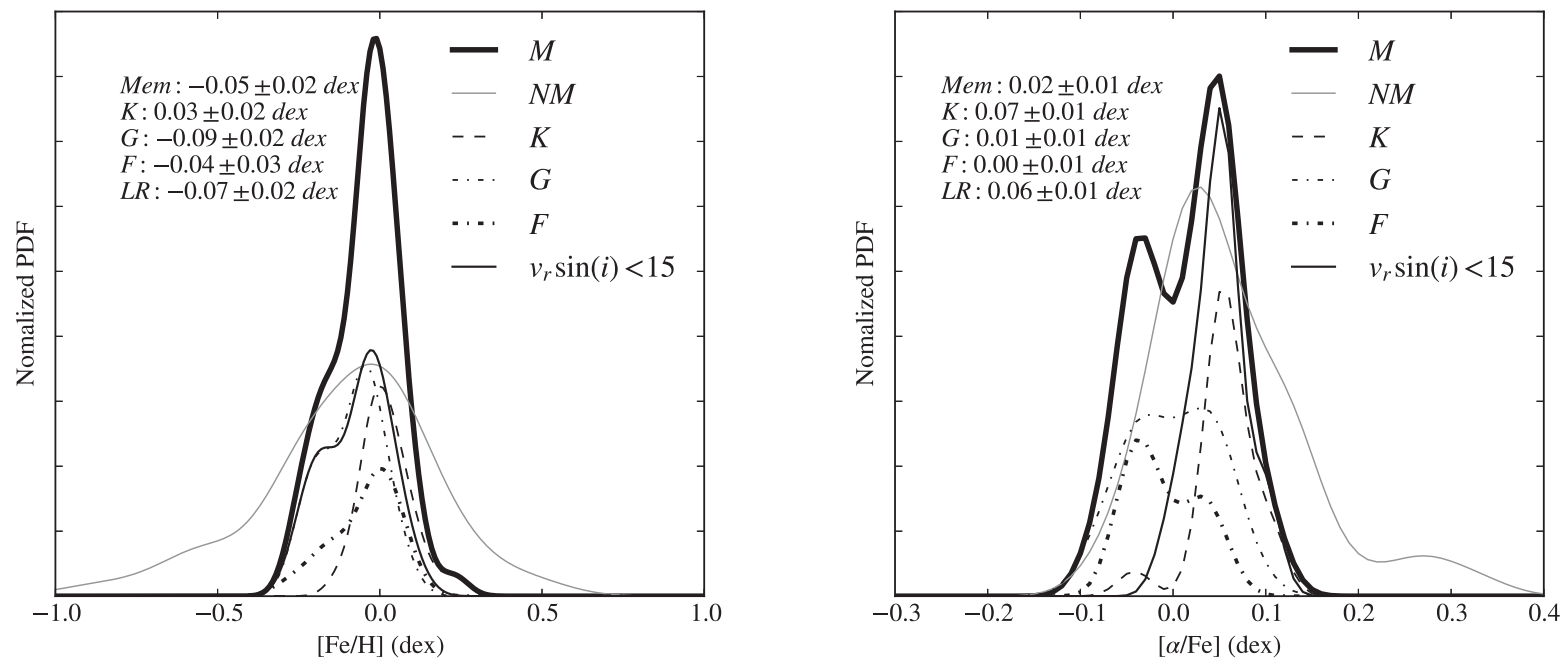

Figure 13. Gaussian kernel density estimates of $[\mathrm{Fe} / \mathrm{H}]$ (left) and $[\alpha / \mathrm{Fe}]$ (right) in NGC 2422 . The thick black line shows the full RV member population and the thin grey line the RV non-member sample. The other lines show the distributions for various subsets of our RV member sample. LR stands for low-rotation and gives the mean for the $v_{r} \sin (i)<15 \mathrm{~km} \mathrm{~s}^{-1} \mathrm{RV}$ member sample.

for systematics in our science targets not present in our standard stars [e.g. due to their uniformly low $v_{r} \sin (i)$ ].

Figs 14 and 15 plot the distribution of stellar rotation with $T_{\text {eff }}$. These figures show uniformly elevated rotation rates in F stars and a general decline with spectral type, but also a spread among K stars. For comparison, we also show the distribution for the similarly aged Pleiades ( $\sim 100 \mathrm{Myr}$, see e.g. Queloz et al. 1998). Our distributions for both clusters appear consistent with the overall trend with $T_{\text {eff }}$ seen in the Pleiades data, though we do not see elevated rotation in $\mathrm{G}$ stars and see a greater spread among K stars.

A simplistic Monte Carlo resampling simulation where we draw with replacement from our sample until we attain our sample size indicates that 14 percent of the time in NGC 2516 and 17 per cent of the time in NGC 2422 we would expect to see no G stars with elevated $v_{r} \sin (i)$ - i.e. above the visual envelope traced by the bulk of stars in the left panels of Figs 14 and 15 - assuming our data samples well the underlying distribution. The Pleiades sample reported in Queloz et al. (1998) has no early to mid K stars with rotation rates above $\sim 20 \mathrm{~km} \mathrm{~s}^{-1}$, at odds with our sample. Comparing our NGC 2516 and NGC 2422 samples, we find that they are in agreement except for early $\mathrm{K}$ stars, where we see many higher rotation stars in the NGC 2516, despite its older age. A Monte Carlo simulation suggests there is only a 9 per cent chance that their early $\mathrm{K}$ distributions are similar. Table 6 reports means and standard deviations for $v_{r} \sin (i)$ for three temperature ranges roughly corresponding to the $\mathrm{F}, \mathrm{G}$ and $\mathrm{K}$ stars in our sample.

We combine our data for $v_{r} \sin (i),[\mathrm{Fe} / \mathrm{H}]$, membership and binarity in Figs 16 and 11, which are colour-magnitude and temperature-magnitude diagrams for our clusters. As previously mentioned, the enhanced metallicity among members hotter than $\sim 6000 \mathrm{~K}$ is likely an artefact of the stars' higher stellar rotation and our fitting approach. The disagreement between $\mathrm{G}$ and $\mathrm{K}$ stars does not follow from this as our K star spectra appear not to suffer from the same optimization issue. 

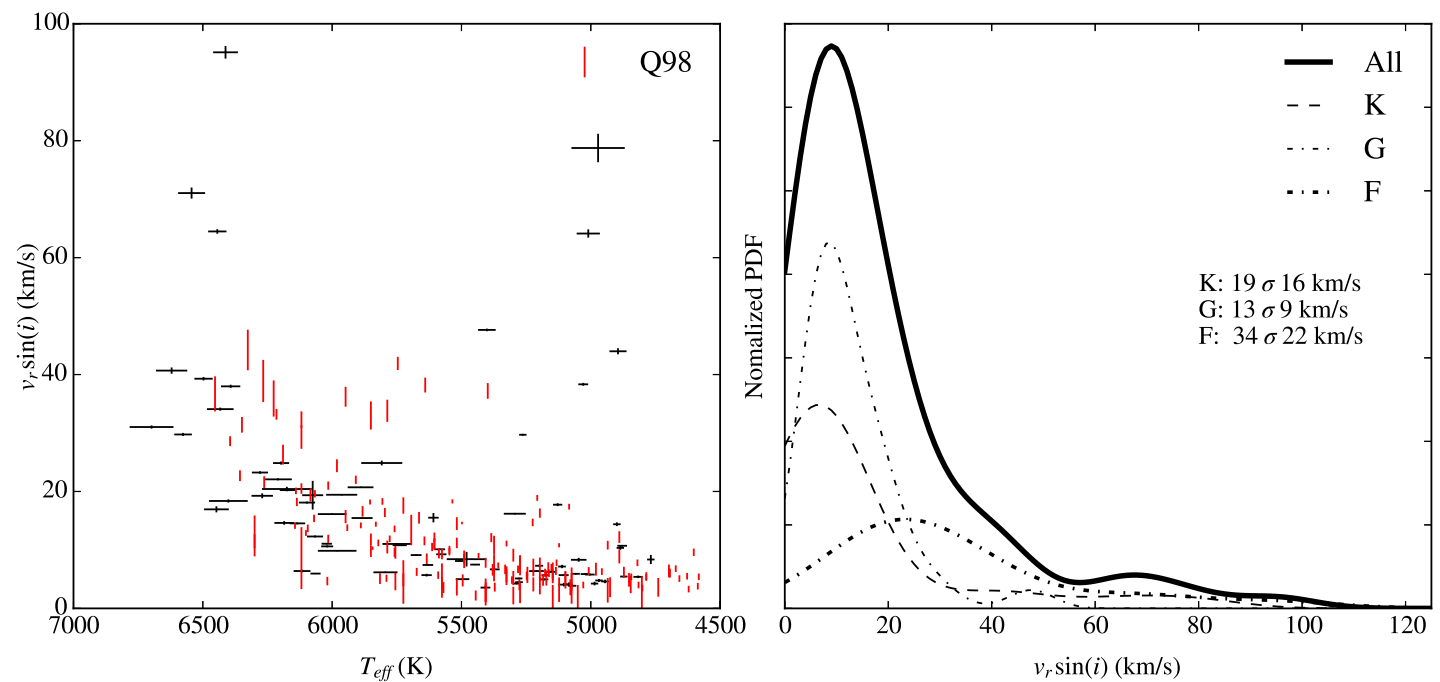

Figure 14. Left: $v_{r} \sin (i)$ values we measure for RV members in NGC 2516 as a function of corrected $T_{\text {eff }}$ along with values for stars in the Pleiades taken from tables 3 and 4 of Queloz et al. (1998) in red. We have used Casagrande et al. (2010) with $[\mathrm{Fe} / \mathrm{H}]=+0.02$ and $E(B-V)=0.04$ to convert colours to temperatures for Pleiades stars. Right: Gaussian kernel density estimates for the RV members grouped by spectral type.
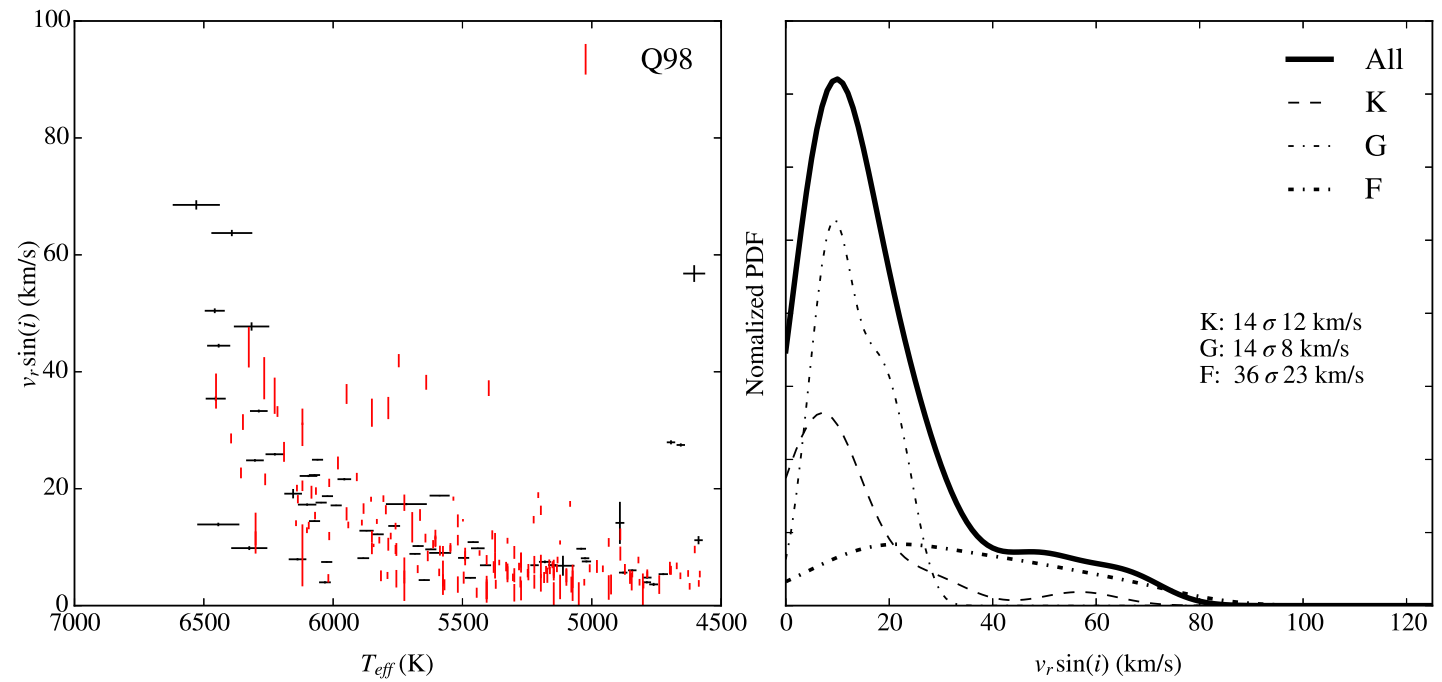

Figure 15. Left: $v_{r} \sin (i)$ values we measure for RV members in NGC 2422 as a function of corrected $T_{\text {eff }}$ along with values for stars in the Pleiades taken from tables 3 and 4 of Queloz et al. (1998) in red. We have used Casagrande et al. (2010) with $[\mathrm{Fe} / \mathrm{H}]=+0.02$ and $E(B-V)=0.04$ to convert colours to temperatures for Pleiades stars. Right: Gaussian kernel density estimates for the RV members grouped by spectral type.

Table 6. Aggregate $v_{r} \sin (i)$ values.

\begin{tabular}{lccc}
\hline Cluster & $\begin{array}{c}T_{\text {eff }} \geq 6100 \mathrm{~K} \\
\left(\mathrm{~km} \mathrm{~s}^{-1}\right)\end{array}$ & $\begin{array}{c}5250 \mathrm{~K} \leq T_{\text {eff }}<6100 \mathrm{~K} \\
\left(\mathrm{~km} \mathrm{~s}^{-1}\right)\end{array}$ & $\begin{array}{c}T_{\text {eff }}<5250 \mathrm{~K} \\
\left(\mathrm{~km} \mathrm{~s}^{-1}\right)\end{array}$ \\
\hline NGC 2516 & $33 \pm 20.9(N=20)$ & $13 \pm 9.0(N=24)$ & $14 \pm 18.0(N=33)$ \\
NGC 2422 & $31 \pm 16.2(N=11)$ & $13 \pm 5.6(N=24)$ & $13 \pm 12.8(N=18)$ \\
Non-members & $31 \pm 26.1(N=25)$ & $5 \pm 1.3(N=44)$ & $5 \pm 5.4(N=44)$ \\
\hline
\end{tabular}

Note. Means and standard deviations for $v_{r} \sin (i)$ measurements of RV members in each of our targeted clusters as well as an aggregate value for all RV non-members. Stars are grouped by measured $T_{\text {eff. }}$.

\section{STELLAR JITTER}

Young stars are well known to exhibit stellar activity (e.g. surface spots and flares) that can induce measurable distortions in line profiles, thereby shifting the recovered RVs from their true values (for a review, see Lagrange et al. 2013). Stars as young as those in the $\sim 140$-Myr- and $\sim 75$-Myr-old clusters we have targeted are often avoided in exoplanet searches for fear of stellar jitter hindering detection of low-amplitude RV variability. Levels in excess of $300 \mathrm{~m} \mathrm{~s}^{-1}$ are seen at ages of a few million years (see fig. 3 of Lagrange et al. 2013) in RVs obtained from optical spectra while others have reported values of $60 \mathrm{~m} \mathrm{~s}^{-1}$ at $\sim 200 \mathrm{Myr}$ (Paulson \& 


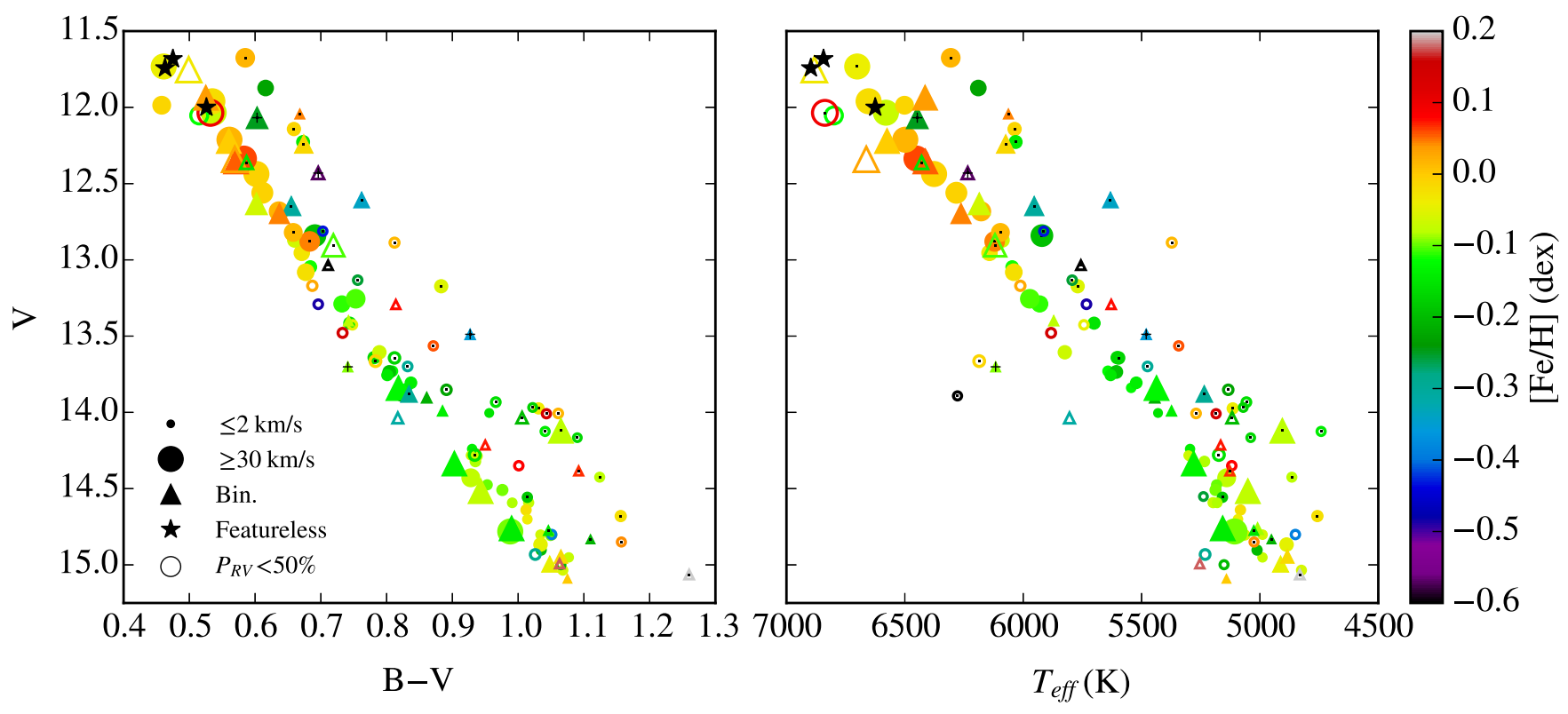

Figure 16. Colour-magnitude (left) and temperature-magnitude (right) diagrams for targets in NGC 2516. Stars we identified as probable members (see Section 5) are shown as filled circles or, for binaries, triangles. Double-lined binaries have a tiny black + on them and targets J01 identifies as photometric binaries a tiny black dot. The colour shows $[\mathrm{Fe} / \mathrm{H}]$ and point $\operatorname{size} v_{r} \sin (i)$. Unfilled points show the same for targets that do not pass our membership test. The filled black stars correspond to the featureless spectra discussed in Section 5.1 and are plotted using $T_{B-V}$. We have corrected the $T_{\text {eff }}$ values of members using equation (2).

Yelda 2006). IR spectra can reduce activity-induced variations by about twofold due to the decreased star-spot contrast $\left(\sim 100 \mathrm{~m} \mathrm{~s}^{-1}\right.$ at $15 \mathrm{Myr}$; Bailey et al. 2012) but this approach is usually hindered by smaller wavelength coverage and noisier detectors.

Stars older than $\sim 500 \mathrm{Myr}$ show significantly reduced activity with levels below $20 \mathrm{~m} \mathrm{~s}^{-1}$ in the optical (Paulson et al. 2004; Quinn et al. 2012, 2014). To date, there is no generally accepted level of jitter as a function of age in the $\sim 30-500 \mathrm{Myr}$ range, though this may be because there is not one. The large spread in stellar rotation and activity exhibited by coeval stars with ages of $\sim 50-300$ Myr may well mean a one-size-fits-all approach is not applicable (Lagrange et al. 2013). In this section, we investigate the level of stellar jitter, $\sigma_{\text {jitter }}$, seen and, despite our comment regarding one-size-fits-all approaches, attempt to determine an average value for each cluster.

In Section 4 , we introduced $\sigma_{\text {stel }}$ to quantify the level of stellar velocity variability we see beyond that explained by our measurement errors alone. There we argued that any variability seen above a $300 \mathrm{~m} \mathrm{~s}^{-1}$ threshold was induced purely by the presence of a stellar companion, neglecting variations caused by stellar activity. As levels at these ages are likely $\lesssim 150 \mathrm{~m} \mathrm{~s}^{-1}$ (Lagrange et al. 2013), that choice did not appreciably affect our binary determinations and allowed us to defer the detailed issue until now. We defined

$\sigma_{\text {stel }} \equiv \sqrt{\sigma_{\text {obs }}^{2}-\overline{\sigma_{\text {meas }}^{2}}}$

where $\sigma_{\mathrm{obs}}^{2}$ is the measurement weighted variance in our RV measurements and $\overline{\sigma_{\text {meas }}^{2}}$ is the mean measurement variance. Here we acknowledge the contribution of jitter explicitly with the approximation that $\sigma_{\text {stel }}^{2}=\sigma_{\text {companion }}^{2}+\sigma_{\text {jitter }}^{2}$ and point out that in the absence of a companion, $\sigma_{\text {stel }}$ serves as a measure of $\sigma_{\text {jitter }}$.

Fig. 17 shows $\sigma_{\text {stel }}^{2}$ versus $v_{r} \sin (i)$ for the different subsets $-\mathrm{RV}$ members and non-members, RV binaries and RV variables - in a $\log -\log$ plot. This plot shows clearly that many stars in our sample exhibit greater variability than one would expect from measurement errors alone. A higher proportion of non-member stars have

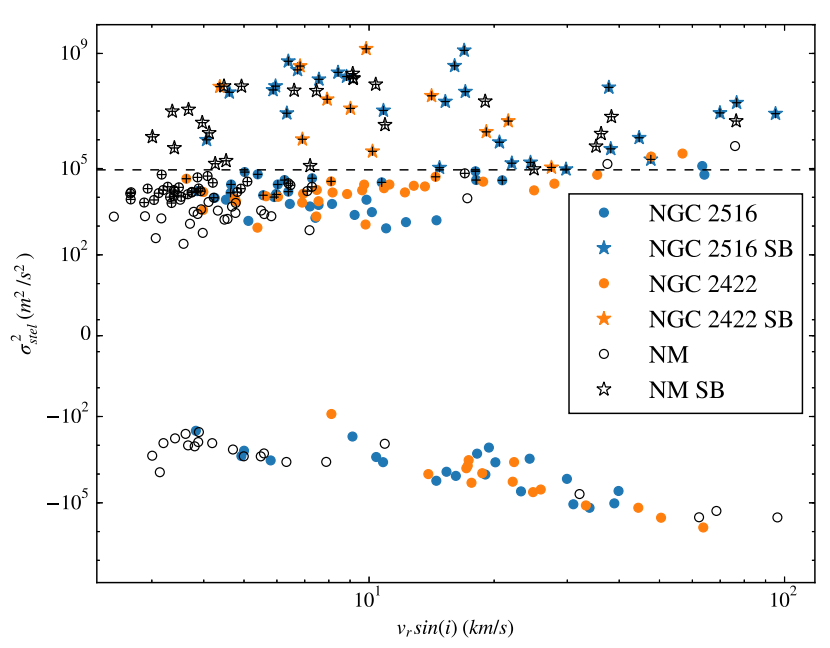

Figure 17. $\sigma_{\text {stel }}^{2}$ versus $v_{r} \sin (i)$ for stars in our sample. Circles denote RV single stars and stars denote RV binary stars. Unfilled markers are targets that failed our membership test. A black cross has been placed on markers that show statistically significant RV variability. Blue marks indicate members of NGC 2516 and orange of NGC 2422. The dashed horizontal like demarcates our RV binary threshold.

a comparable (or greater) $\sigma_{\text {meas }}$ than $\sigma_{\text {obs }}$, as might be expected in a population composed of older field dwarfs and giants and hence subject to less stellar activity (e.g. Johnson et al. 2010). The frequency of stars with a negative $\sigma_{\text {stel }}^{2}$ and $v_{r} \sin (i)<8 \mathrm{~km} \mathrm{~s}^{-1}$ is consistent with our simulated data set. At higher rotation rates, we see more stars with a variance deficit, consistent with our mean RV prior biasing our results towards non-variability.

We employ two different approaches to estimate a typical value for $\sigma_{\text {jitter }}$ in each cluster. The first is to ask what the mean level of stellar variability is in our sample $\left(\sqrt{\overline{\sigma_{\text {stel }}^{2}}}\right)$. The second and more 

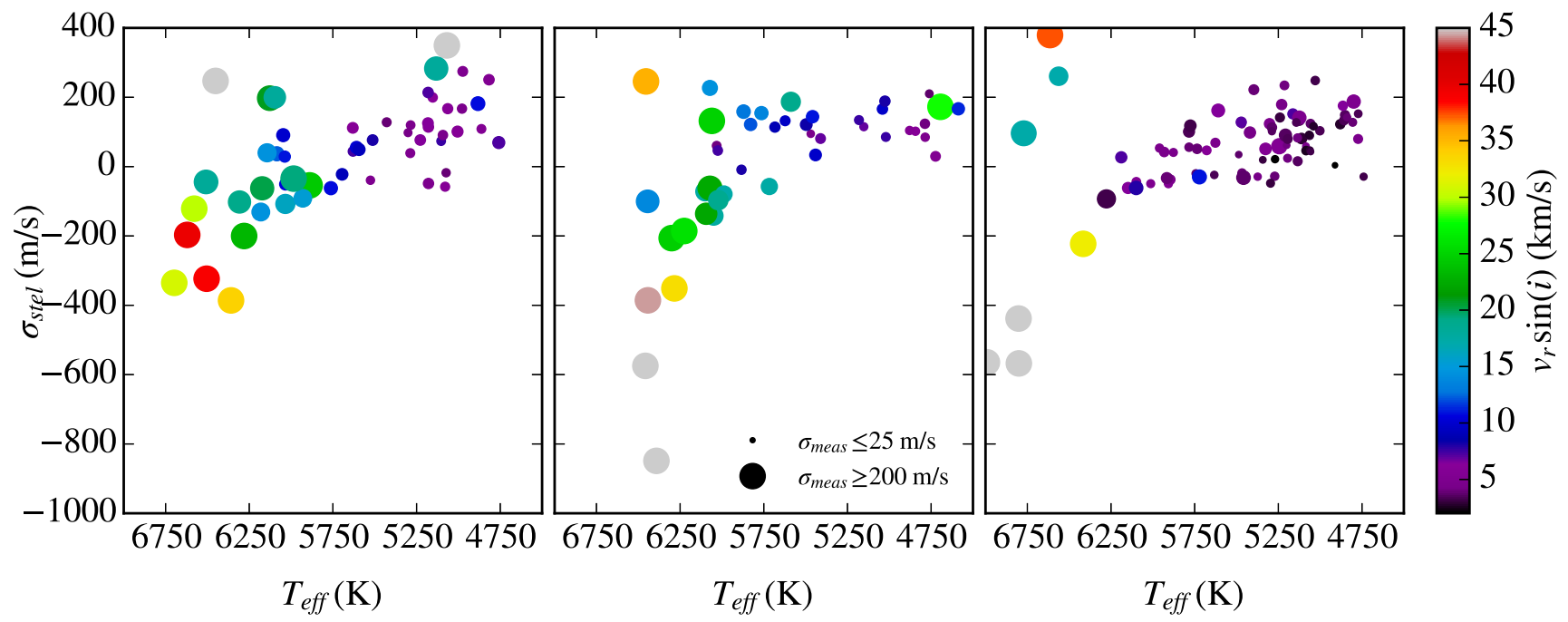

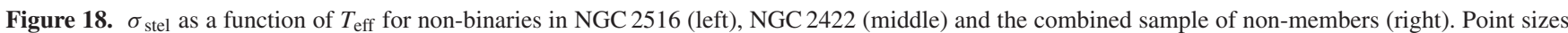
are scaled by the mean measurement error for each target and point colour is used to show $v_{r} \sin (i)$. We plot negative values for $\sigma_{\text {stel }}$ by computing the root of the absolute value of the variance and preserving the sign. The large number of rapidly rotating stars with negative $\sigma_{\text {stel }}$ is a result of our optimizer exhibiting a strong preference for the initial RV in spectra with broad features. Based on this figure, we adopt the $T_{\text {eff }}<5800 \mathrm{~K}$ and $v_{r} \sin (i)<15 \mathrm{~km} \mathrm{~s}^{-1}$ sample to study stellar activity in our sample.

Table 7. Stellar jitter.

\begin{tabular}{llll}
\hline Method & NGC 2516 & $\begin{array}{c}\sigma_{\text {jitter }}\left(\mathrm{m} \mathrm{s}^{-1}\right) \\
\text { NGC 2422 }\end{array}$ & Non-members \\
\hline 1 & $48 \pm 29(14)$ & $131 \pm 44(18)$ & $90 \pm 31(45)$ \\
2 & $77 \pm 10(18)$ & $138 \pm 10(19)$ & $56 \pm 7(30)$ \\
Averages & $74 \pm 9$ & $138 \pm 2$ & $58 \pm 7$ \\
\hline
\end{tabular}

Note. Numbers in parentheses report sample size. An additional $10 \mathrm{~m} \mathrm{~s}^{-1}$ has been added to correct for the underestimate seen in our simulations.

involved method is to assume that each star's set of RV measurements is drawn from Gaussians at each star's absolute RV with widths $\sigma^{2}=\sigma_{\text {meas }}^{2}+\sigma_{\text {stel }}^{2}$, then we determine the likelihood function for $\sigma_{\text {stel }}$ given our data and solve for $\sigma_{\text {stel }}$ with maximum likelihood. In both cases, we must consider the $\sigma_{\text {companion }}$ contribution of genuine companions; recall from Section 5.2 that we expect at least 37 per cent of stars with $\sigma_{\text {stel }}<300 \mathrm{~m} \mathrm{~s}^{-1}$ to be long-period binaries. We account for companions in our sample in two ways. First, we compute the mean or expectation value of $\sigma_{\text {stel }}$ iteratively, excluding any stars with $a \geq 99$ per cent chance of being a variable $\left(P_{\mathrm{v}} \geq 0.99\right.$; Section 8$)$ in the presence of the jitter inferred from the previous iteration. Secondly, we apply both tests to our simulated population (Section 4.1) in the presence of added Gaussian jitter. We find that each method recovers a jitter estimate $\sim 10 \mathrm{~m} \mathrm{~s}^{-1}$ under the input value and that the two methods agree at the $\mathrm{m} \mathrm{s}^{-1}$ level.

We use RV single members $\left(\sigma_{\text {stel }}<300 \mathrm{~m} \mathrm{~s}^{-1}\right)$ with a corrected $T_{\text {eff }}<5800 \mathrm{~K}$ and $v_{r} \sin (i)<15 \mathrm{~km} \mathrm{~s}^{-1}$ (Fig. 18) in NGC 2516 $(N=30)$ and NGC $2422(N=20)$ to estimate the level of stellar activity in our targets. In practice, these temperature and rotation cuts are equivalent to a measurement error cut of $\sigma_{\text {meas }}<100 \mathrm{~m} \mathrm{~s}^{-1}$, which we use when testing our approach with simulated data. The two tests we perform are described in the following paragraphs and the results are listed in Table 7. Fig. 19 shows the starting and final samples for method 2. The set of targets used is similar for the first method. Although we observe significant variability in our non-member sample, we argue that the poorer accuracy of the stellar templates used for these fits (i.e. very inaccurate $\log (g)$ values and hence poorly optimized $T_{\text {eff }}$ and $[\mathrm{Fe} / \mathrm{H}]$ ) may mean that we underestimate our measurement errors. We also note that the jitter we recover is about the same as our median value for $\sigma_{\text {meas }}$.

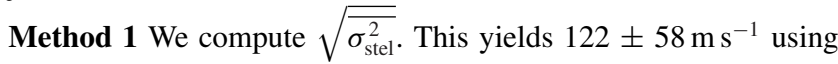
30 stars in NGC 2516, which converges to $38 \pm 29 \mathrm{~m} \mathrm{~s}^{-1}(N=14)$ after iteratively excluding probable variables. In NGC 2422, we measure a value of $130 \pm 50 \mathrm{~m} \mathrm{~s}^{-1}$ using 20 stars, which converges to $121 \pm 44 \mathrm{~m} \mathrm{~s}^{-1}(N=18)$ after iteratively excluding stars with $P_{\mathrm{v}} \geq 0.99$. Our simulation suggests that these values systematically underestimate the true variability by $10 \mathrm{~m} \mathrm{~s}^{-1}$.

Method 2 We assume that our RVs $\left(x_{i j}\right)$ are drawn from a set of Gaussians each located at a star's relative motion along the line of sight $\left(\mu_{i}\right)$ and a standard deviation corresponding to the quadrature sum of $\sigma_{\text {meas }, i}$ and $\sigma_{\text {stel }}$ :

$\left(x_{11}, x_{i j}, \ldots, x_{N M_{i}}\right) \sim N\left(\mu_{i}, \sqrt{\sigma_{\text {meas }, i}^{2}+\sigma_{\text {stel }}^{2}}\right)$.

The likelihood function for which is

$$
\begin{aligned}
& f_{\sigma_{\text {stel }}, \mu_{1}, \ldots, \mu_{N}}\left(\mathbf{X}_{\mathbf{1}}, \ldots, \mathbf{X}_{\mathbf{N}}\right) \\
& =\prod_{i=1}^{N} \prod_{j=1}^{M_{i}} \frac{1}{\sqrt{2 \pi\left(\sigma_{\text {meas }, i}^{2}+\sigma_{\text {stel }}^{2}\right)}} e^{-\frac{x_{i j}-\mu_{i}}{2\left(\sigma_{\text {meas }, i}^{2}+\sigma_{\text {stel }}^{2}\right)}} .
\end{aligned}
$$

Noting that

$$
\frac{1}{M_{i}} \sum_{j=1}^{M_{i}}\left(x_{i j}-\overline{x_{i}}\right)^{2} \approx \sigma_{\mathrm{obs}, i}^{2}
$$

and that $\mu_{i} \approx \overline{x_{i}}$, we can take the $\log$ and write the log-likelihood as

$$
\begin{aligned}
l\left(\sigma_{\text {stel }}\right)= & -\frac{1}{2} \sum_{i=1}^{N} M_{i} \\
& \times\left(\frac{\sigma_{\text {obs }, i}^{2}}{\sigma_{\text {meas }, i}^{2}+\sigma_{\text {stel }}^{2}}+\log \left(2 \pi\left(\sigma_{\text {meas }, i}^{2}+\sigma_{\text {stel }}^{2}\right)\right)\right) .
\end{aligned}
$$



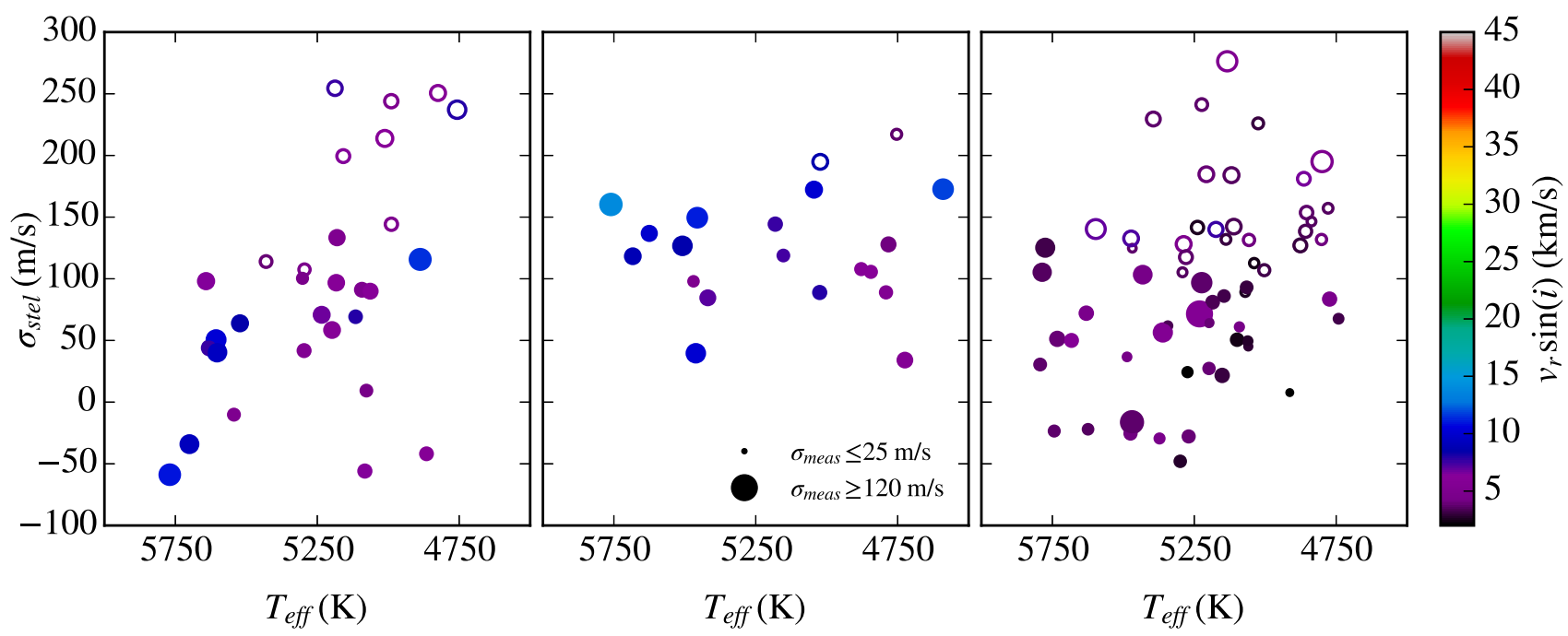

Figure 19. Starting and ending samples of $\sigma_{\text {stel }}$ used to estimate stellar activity levels in NGC 2516 (left), NGC 2422 (middle) and the combined sample of non-members (right) with method 2 (method 1 is similar). Point sizes are scaled by the mean measurement error for each target and point colour is used to show $v_{r} \sin (i)$. Open circles denote targets that were excluded by the $P_{\mathrm{v}} \geq 0.99$ cut when iterating. Negative values for $\sigma_{\text {stel }}$ are the root of the absolute value of the variance with the sign preserved.

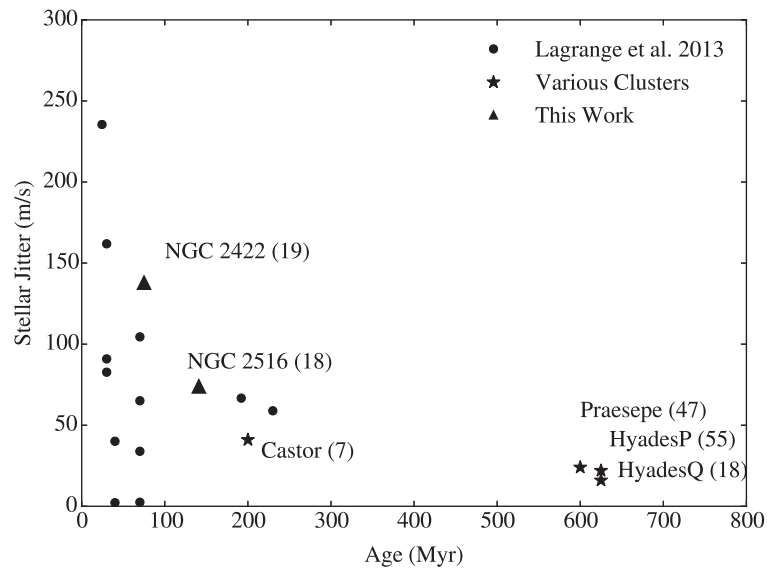

Figure 20. Determinations of stellar jitter for a number of single stars (circles) from Lagrange et al. (2013), averages from stars in open clusters and young associations from Paulson \& Yelda (2006), Paulson et al. (2004) and Quinn et al. (2012, 2014) (stars), and our results in NGC 2516 and NGC 2422 (triangles). For clusters and associations, we note the name and number of stars used in the average.

Here $N$ is the number of stars under consideration and $M_{i}$ the number of observations of star $i$. The peak of this function then provides an estimate of typical $\sigma_{\text {stel }}$ for the sample of stars, which after iterating should be predominately driven by companion-free (or effectively so) stars. We find $\sigma_{\text {stel }}=130 \pm 10 \mathrm{~m} \mathrm{~s}^{-1}$ using 30 stars in NGC 2516, which converges to $67 \pm 10 \mathrm{~m} \mathrm{~s}^{-1}(N=18)$ after iteratively excluding stars with $P_{\mathrm{v}}>0.99$. In NGC 2422, we measure a value of $135 \pm 10 \mathrm{~m} \mathrm{~s}^{-1}$ using 20 stars, which converges to $128 \pm 10 \mathrm{~m} \mathrm{~s}^{-1}(N=19)$. Our simulation suggests that these values systematically underestimate the true variability by $10 \mathrm{~m} \mathrm{~s}^{-1}$. Table 7 also reports values for the non-member population.

In Fig. 20, we show the levels of jitter we measure in NGC 2516 and NGC 2422 alongside measurements of stellar jitter in single stars from Lagrange et al. (2013, measured using HARPS at $\sim 530 \mathrm{~nm}$ ) and the values determined by various other authors (also in the optical) for a number of open clusters and associations. Our results in both clusters are generally consistent with existing results: large scatter when young, decreasing to $\lesssim 150 \mathrm{~m} \mathrm{~s}^{-1}$ by $\sim 100 \mathrm{Myr}$. Note that our observed rotation rates in NGC 2422 (Table 6) are consistent with those in NGC 2516 but the binary fraction in NGC 2516 is far higher, suggesting that neither stellar rotation nor binarity are to blame for NGC 2422's higher activity. In both clusters, and NGC 2516 especially, we measured a wide range of $\sigma_{\text {stel }}$ in our nonvariable population, suggesting there is, indeed, a large spread in activity-induced RV variability among individual stars as was previously seen at $\sim 50-75 \mathrm{Myr}$ in the work of Lagrange et al. (2013) and shown in Fig. 20.

\section{STELLAR VARIABILITY}

We now turn our attention to identifying which stars in our sample exhibit statistically significant RV variability and to placing limits on companion masses for our entire sample. Our goal here is to provide a refined pool of targets that merit following up with some combination of single-object RV spectroscopy and multi-band photometric monitoring to assess whether stellar surface activity is responsible for the variability we see.

We compute the raw probability that each star is an RV variable and the probability that each star is an RV variable in the presence of the appropriate $\sigma_{\text {jitter }}$ from Table 7. This probability, $\mathrm{P}_{\mathrm{v}}=\mathrm{P}\left(\mathrm{X}<\chi^{2}\right)$, is computed using the RVs and $\sigma_{\text {meas }}$ values of each star, in the latter case incorporating $\sigma_{\text {jitter }}$ by adding in quadrature with $\sigma_{\text {meas }}$. In both cases, we exclude RV outliers $>3 \sigma_{\text {obs }}$ from the mean RV. This excludes a single epoch for 42 stars and two epochs in another two stars for a total of $44 \mathrm{RVs}$ across our sample of $\sim 2500$ observations. The former raw $P_{v}$ then is an estimate of the likelihood that each star exhibits any sort of velocity variability while the latter estimates the probability that variations are induced by a companion. These probabilities are reported in Tables $\mathrm{A} 1$ and $\mathrm{A} 2$.

We performed the same analysis on our simulated RVs to estimate the false-positive and false-negative rates for stellar and exoplanetary companions as a function of $P_{v}$ cut and gauge our companion sensitivity. Figs 21 and 22 show our false-positive and false-negative 

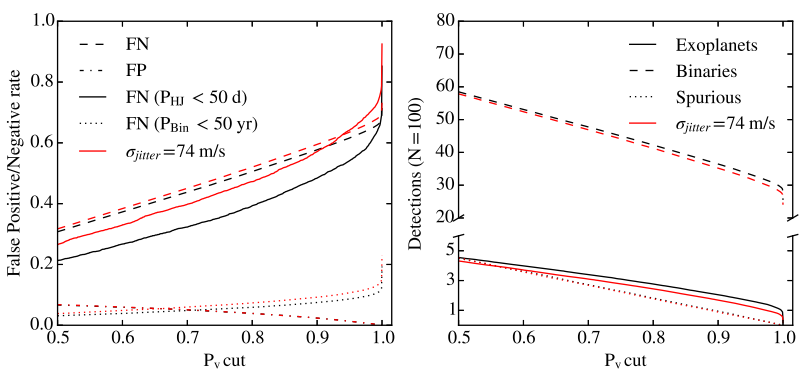

Figure 21. Left: False-positive (FP) and false-negative (FN) rates of our variability test for binaries and exoplanets in our companion simulation for NGC 2516 both without (black) and with (red, styles the same as for black lines) additional stellar variability. Right: The number of true binaries (not merely binaries with $\sigma_{\text {stel }}>300 \mathrm{~m} \mathrm{~s}^{-1}$ ), exoplanet-hosting and spuriously variable stars we would expect to flag in a sample of 100 stars, again shown without (black) and with (red) additional stellar variability. This simulation was run assuming a binary fraction of 85 per cent consistent with J01 and assuming the cadence of our 12 observations in NGC 2516. Though not shown, we note a binary fraction of 100 per cent would increase the expected number of binary detections by approximately seven, roughly independent of $P_{v}$ cut.
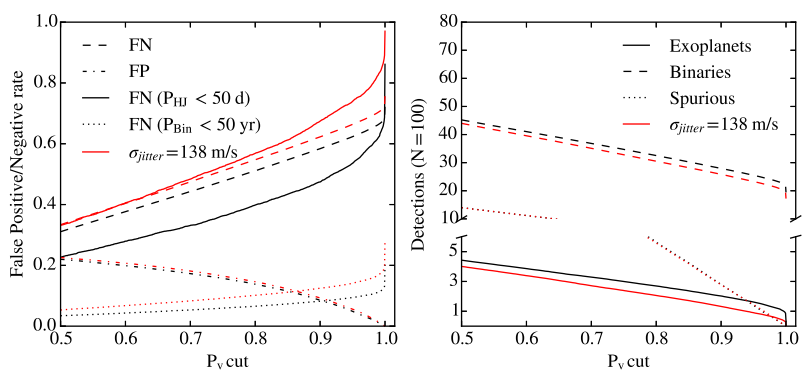

Figure 22. Left: False-positive (FP) and false-negative (FN) rates of our variability test for binaries and exoplanets in our companion simulation for NGC 2422 both without (black) and with (red, styles the same as for black lines) additional stellar variability. Right: The number of true binaries (not merely binaries with $\sigma_{\text {stel }}>300 \mathrm{~m} \mathrm{~s}^{-1}$ ), exoplanet-hosting and spuriously variable stars we would expect to flag in a sample of 100 stars, again shown without (black) and with (red) additional stellar variability. This simulation was run assuming a binary fraction of 62 per cent and assuming the cadence of our ten observations in NGC 2422.

rates as a function of $P_{v}$ along with the number of exoplanets, binaries and false variables we would expect in a population of 100 stars for each of our clusters. This indicates that for a $P_{v}$ cut of 0.96 , we can expect a roughly equivalent number of exoplanet hosts as false positives while being sensitive to 92 per cent of binaries with $P \lesssim 50 \mathrm{yr}$ and $\sim 40$ per cent of simulated exoplanets with $P<50 \mathrm{~d}$. Assuming NGC 2516 has a binary fraction of 85 per cent, consistent with $\mathrm{J} 01$, and a jitter of $74 \mathrm{~m} \mathrm{~s}^{-1}$, our simulations predict RV binaries $\left(\sigma_{\text {stel }}>300 \mathrm{~m} \mathrm{~s}^{-1}\right)$ to be composed of 99 per cent stellar multiples, 0.85 per cent exoplanets and 0.15 per cent false positives. Low-amplitude variables should be composed of 84 per cent stellar, 12 per cent exoplanets and 4 per cent false positives. In NGC 2422, we adopt a binary fraction of 62 percent and predict binaries to be composed of 97.6 per cent stellar, 1.4 per cent exoplanets and 1 percent false positives. Our single sample should consist of 67 percent binaries, 12 percent exoplanets and 21 percent false positives.
Table 8. Number of RV variables stars in NGC 2516 and NGC 2422

\begin{tabular}{lcccc}
\hline & \multicolumn{2}{c}{ NGC 2516 sample } & \multicolumn{2}{c}{ NGC 2422 sample } \\
Non- \\
Group & Members & Nembers & Members & $\begin{array}{c}\text { Non- } \\
\text { members }\end{array}$ \\
\hline Usable targets & 81 & 41 & 57 & 65 \\
RV variables & 52 & 26 & 35 & 47 \\
RV variables including $\sigma_{\text {jitter }}$ & 43 & 23 & 14 & 35 \\
SB2 & $2 / 3(2)$ & $1 / 1(1)$ & $2 / 2$ & $1 / 2$ \\
$\sigma_{\text {stel }} \geq 300 \mathrm{~m} \mathrm{~s}^{-1}$ & $16 / 27(10)$ & $8 / 13(7)$ & $7 / 11$ & $7 / 11$ \\
$\sigma_{\text {stel }}<300 \mathrm{~m} \mathrm{~s}^{-1}$ & $2 / 16(4)$ & $1 / 10(8)$ & $0 / 3$ & $6 / 24$
\end{tabular}

Note. This is a breakdown of RV variability in our sample. Usable targets exclude the five featureless stars, the one star in NGC 2516 for which no usable data were obtained and the one mistakenly targeted early-type star in NGC 2422 (Section 5.1). The first of each pair of numbers is the number of RV variables with at least a 95 percent significant periodogram peak (Section 8.1). This number is followed by the total number of statistically significant $\left(P_{\mathrm{v}}>0.96\right) \mathrm{RV}$ variables, accounting for a jitter level of $74 \mathrm{~m} \mathrm{~s}^{-1}$ in NGC 2516, $138 \mathrm{~m} \mathrm{~s}^{-1}$ in NGC 2422 and $58 \mathrm{~m} \mathrm{~s}^{-1}$ in the field. Numbers in parentheses denote the number of stars in common with photometric binaries reported by J01. Sections 5 and 8 describe the expected make-up of these groups in terms of multiples, long-period multiples, exoplanets and false positives. Tables 9 and 10 provide details of the variables in NGC 2516 and NGC 2422.

\subsection{RV variables}

Adopting a $P_{v}$ threshold of 0.96 , we report the number of significant RV variables we see in Table 8 after accounting for the average $\sigma_{\text {jitter }}$ from Table 7. 53 of our single stars exhibit significant variability after accounting for stellar jitter.

Using a binary fraction of 100 percent in NGC 2516, $\sigma_{\text {jitter }}=74 \mathrm{~m} \mathrm{~s}^{-1}$, our observation spacing and our adopted threshold of $P_{\mathrm{v}}=0.96$, we would expect 39 per cent of members to exhibit significant variability. We observe a significantly higher total variability rate of $53 \pm 6$ percent. Our simulation shows we would expect about 50 per cent for the total variability rate if the true value for $\sigma_{\text {jitter }}$ were in agreement with NGC 2422. As we used only stars with $T_{\text {eff }}<5800 \mathrm{~K}$ and $v_{r} \sin (i)<15 \mathrm{~km} \mathrm{~s}^{-1}$ when computing $\sigma_{\text {jitter}}$, one possibility is that hotter or more rapidly rotating stars make up the majority of these variables. However, that is not the case. Only three of the 16 low-amplitude variables are hot enough or fast enough rotators to be affected by this cut; excluding them lowers our total variability to 49 per cent. Additional data (e.g. activity indicators) or targeted monitoring is needed to address the source of this disagreement.

In NGC 2422, with a binary fraction of 62 percent and $\sigma_{\text {jitter }}=138 \mathrm{~m} \mathrm{~s}^{-1}$, we would expect 25 percent of members to exhibit significant variability. We find a total variability rate of $25 \pm 6$ per cent, in good agreement with our expectations.

We used the GATSPY package (VanderPlas \& Ivezić 2015) to compute Lomb-Scargle periodograms and estimate their significance via a bootstrap simulation for all of our RV variable stars. Of the 62 binaries, 38 exhibit periodogram peaks with $>95$ per cent significance and have periods ranging from just over a day (our data suffers from a strong alias below $\sim 1.1 \mathrm{~d}$ ) to $\sim 3.5 \mathrm{yr}$. Nine RV single stars also exhibited significant peaks with seven between 1.5 and $5.8 \mathrm{~d}$, one at $21 \mathrm{~d}$ and one at $27 \mathrm{~d}$. We then used systemic2 (Meschiari et al. 2012) to fit trial orbits to each of these stars and perform MCMC simulations to estimate possible companion masses and refine the orbital period. Fig. 23 shows the refined periods as a function of $\sigma_{\text {obs }}$ as well as the median $M \sin (i)$ against the refined periods. This draws into stark relief the larger than expected number of exoplanet 

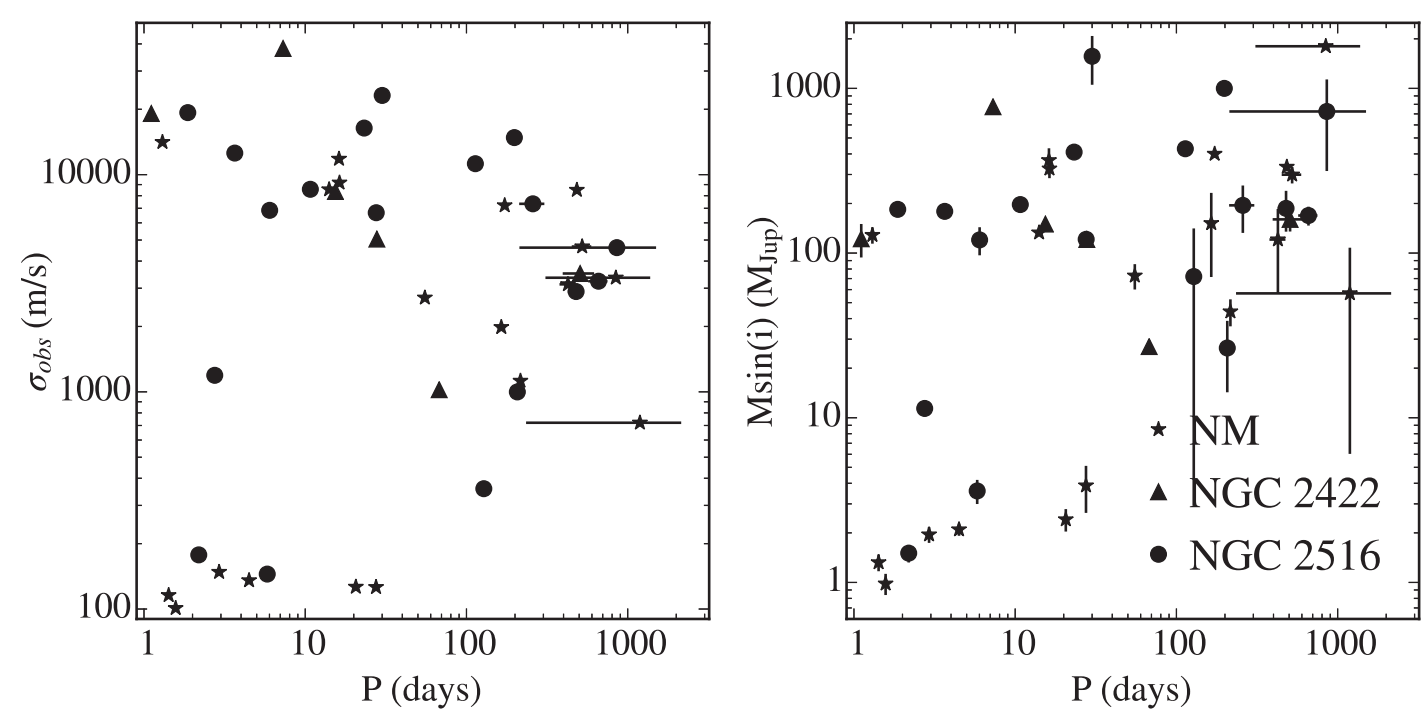

Figure 23. Left: Observed RV standard deviation versus median MCMC period. Right: Inferred companion $M \sin (i)$ versus period. Host star masses were computed using the mass-radius relation of Torres et al. (2010) along with our fitted $T_{\text {eff }}$ (corrected for members by equation 2), [Fe/H] and our adopted log $(g$ ). The masses may, therefore, be significantly off for any non-member giant stars in our sample.

host candidates in our sample, especially for non-member stars. Assuming 1.2 per cent of stars in our sample possess a hot Jupiter companion, we would predict a 1 in 4 chance of there being three in our sample of 81 (two real and one spurious per our false alarm predictions) and expect about a 1 in 10 chance of there being three with one false alarm in our field sample.

The figures in Appendix B (online only) show RV time series, periodograms and - for variables with a significant peak $\gtrsim 1.1 \mathrm{~d}$ - phased RV curves. We stress that these periods should be considered speculative without follow-up observations. Points shown as a red $\mathrm{X}$ were excluded for being $>3 \sigma$ outliers. References to these figures are provided in Tables 9 and 10. These are subsets of our full results table, which consists of key values for RV variables in NGC 2516 and NGC 2422. NGC 2516 has undergone extensive $\mathrm{X}$-ray study and we use the captions of the individual plots to mention any known X-ray variability from D03 or Wolk et al. (2004). For comparison, we also show a selection of RV time series for six non-variable stars in Fig. 24, the properties of which are summarized in Table 11.

\subsection{Short-period companion detection limits}

We determined companion-mass detection limits via Monte Carlo simulations at representative orbital periods of 3,10 and $20 \mathrm{~d}$. The $3 \mathrm{~d}$ period is chosen to be near the peak in the orbital period distribution of hot Jupiters (Winn \& Fabrycky 2015) while the other periods are chosen to demonstrate how the sensitivity declines with increasing orbital period, given the precision and temporal sampling of our survey. At each period, we used our companion detectability simulations (Section 4.1), including the appropriate mean $\sigma_{\text {jitter }}$ $\left(74 \mathrm{~m} \mathrm{~s}^{-1}\right.$ in NGC 2516, $138 \mathrm{~m} \mathrm{~s}^{-1}$ in NGC 2422 and $58 \mathrm{~m} \mathrm{~s}^{-1}$ for non-members) to determine the fraction of orbits for which we would recover $P_{\mathrm{v}}>0.96$. We simulated 2500 orbits per Jupitermass interval over the region from approximately half detectable to 95 per cent detectable and then carried the simulation as far beyond; e.g. a star with $\sim 50$ per cent of companions detectable at $10 \mathrm{M}_{\text {Jup }}$ and $\sim 95$ per cent detectable at $30 \mathrm{M}_{\text {Jup }}$ would have 100000 orbits simulated from 10 to $50 \mathrm{M}_{\text {Jup }}$. This resulted in between 75000 and 1.45 million orbits (mean 120000) simulated for each star and en- sured the parameter space around the 95 per cent threshold was well sampled without requiring excessive simulation time. We then fitted each detected fraction with an incomplete gamma function and adopted the point at which our fit crossed the 95 per cent detectable threshold as our companion-mass limit. We used the mass-radius relation of Torres, Andersen \& Giménez (2010) along with our adopted $T_{\text {eff }},[\mathrm{Fe} / \mathrm{H}]$ and $\log (g)$ to determine a mass for the primary.

The resulting mass limits are reported in Tables A1 and A2. On average for non-variable RV members, we are able to exclude companions greater than $7.4,11.3$ and $23 \mathrm{M}_{\text {Jup }}$ in NGC 2516 and 9.5, 14.4 and $26.1 \mathrm{M}_{\text {Jup }}$ in NGC 2422 at periods of 3, 10 and $20 \mathrm{~d}$. For RV non-members, we exclude companions at 7.3, 11.2 and $21.4 \mathrm{M}_{\mathrm{Jup}}$ on average. These thresholds are heavily influenced by the subset of our sample, which has large measurement errors: the same measures using only stars with $\sigma_{\text {meas }}<150 \mathrm{~m} \mathrm{~s}^{-1}$ are $\sim 50$ per cent lower. Indeed, for approximately $1 / 3$ of our nonvariable stars, we can exclude $3 \mathrm{~d}$ companions more massive than $3 \mathrm{M}_{\text {Jup }}$.

\section{SUMMARY}

We have presented the first results of our survey of Sun-like stars in the core $0.5^{\circ}$ fields of NGC 2516 and NGC 2422. We constructed spectral models of approximately 2700 high-spectral-resolution $(R \sim 50000)$ observations at 7165-7295 $\mathrm{A}$ made with the M2FS on the Clay telescope at Las Campanas Observatory. We used telluric absorption features as an absolute, simultaneous wavelength reference. Each spectrum was modelled as the combination of a telluric spectrum and a synthetically generated stellar spectrum, convolved by a parametrized instrumental profile and projected on to a pixel-space grid.

We used our modelling approach (described fully in B16) to make determinations of RVs, $T_{\text {eff }},[\mathrm{Fe} / \mathrm{H}],[\alpha / \mathrm{Fe}]$ and $v_{r} \sin (i)$ for nearly all 126 and 125 stars in our NGC 2516 and NGC 2422 samples along with estimates for total cluster binary fractions of $100_{-15}^{+0}$ per cent and $62 \pm 16$ per cent. Of the surveyed stars, 81 and 57 proved to be RV members, 12 of which were not previously identified by photometry in NGC 2422. We also noted the presence of a RV clustering of 11 stars along a line of sight to the Monoceros stream 
Table 9. Variables in NGC 2516.

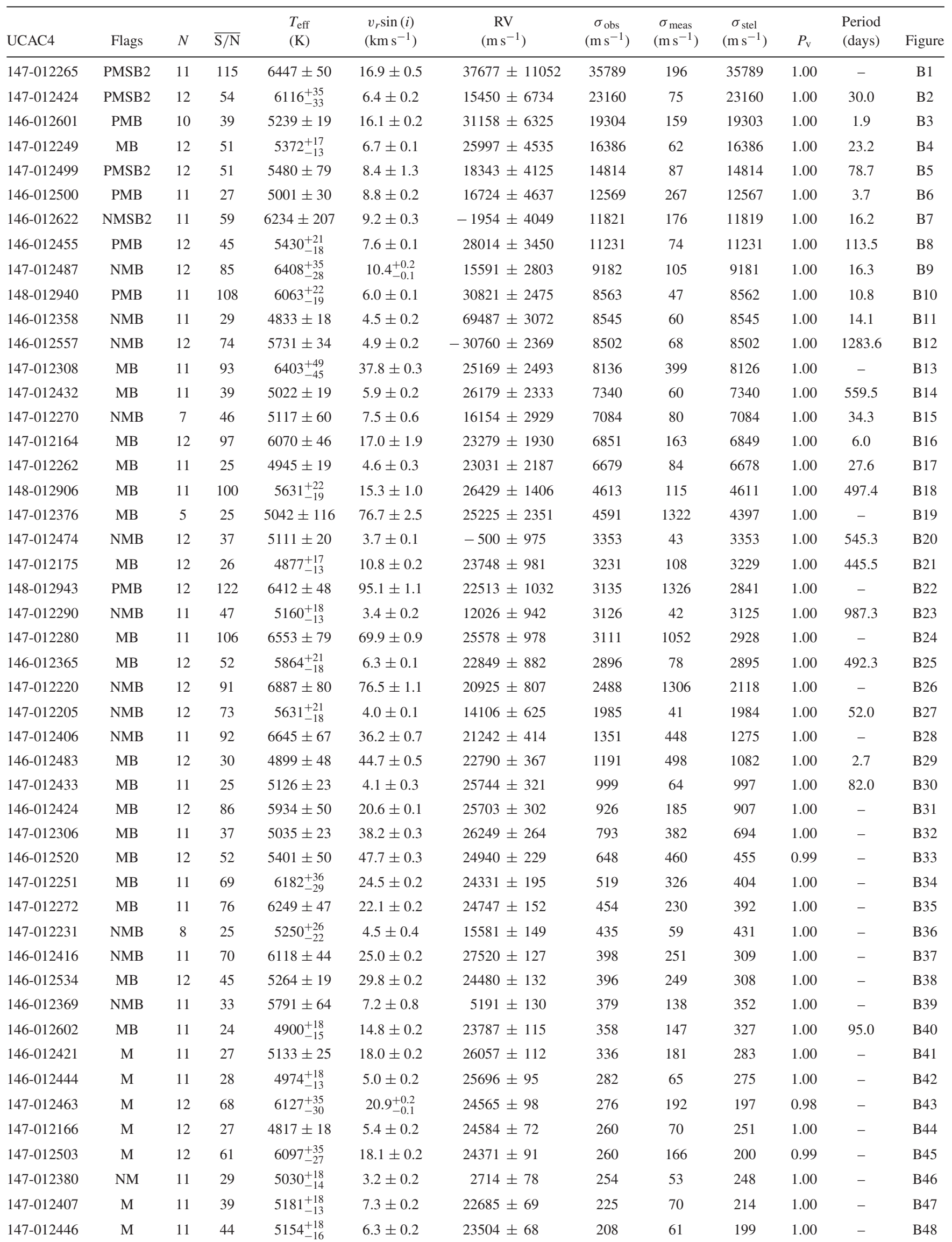


Table 9 -continued

\begin{tabular}{|c|c|c|c|c|c|c|c|c|c|c|c|c|}
\hline UCAC4 & Flags & $\mathrm{N}$ & $\overline{\mathrm{S} / \mathrm{N}}$ & $\begin{array}{l}T_{\text {eff }} \\
(\mathrm{K})\end{array}$ & $\begin{array}{c}v_{r} \sin (i) \\
\left(\mathrm{km} \mathrm{s}^{-1}\right)\end{array}$ & $\begin{array}{c}\mathrm{RV} \\
\left(\mathrm{m} \mathrm{s}^{-1}\right)\end{array}$ & $\begin{array}{c}\sigma_{\mathrm{obs}} \\
\left(\mathrm{m} \mathrm{s}^{-1}\right)\end{array}$ & $\begin{array}{c}\sigma_{\text {meas }} \\
\left(\mathrm{m} \mathrm{s}^{-1}\right)\end{array}$ & $\begin{array}{c}\sigma_{\text {stel }} \\
\left(\mathrm{m} \mathrm{s}^{-1}\right)\end{array}$ & $P_{\mathrm{V}}$ & $\begin{array}{l}\text { Period } \\
\text { (days) }\end{array}$ & Figure \\
\hline $147-012375$ & M & 12 & 30 & $4883 \pm 25$ & $10.7 \pm 0.2$ & $25355 \pm 61$ & 207 & 99 & 182 & 1.00 & - & B49 \\
\hline $147-012307$ & M & 11 & 30 & $5064_{-14}^{+18}$ & $6.0 \pm 0.2$ & $24607 \pm 60$ & 180 & 67 & 167 & 1.00 & - & B51 \\
\hline $146-012532$ & M & 11 & 30 & $4979_{-14}^{+18}$ & $4.7 \pm 0.2$ & $24935 \pm 57$ & 178 & 60 & 167 & 1.00 & 2.2 & B52 \\
\hline $147-012428$ & $\mathrm{NM}$ & 12 & 34 & $5240 \pm 18$ & $2.9 \pm 0.2$ & $74928 \pm 49$ & 153 & 58 & 142 & 1.00 & - & B55 \\
\hline $146-012330$ & M & 9 & 28 & $5182_{-15}^{+20}$ & $6.4 \pm 0.2$ & $24284 \pm 51$ & 145 & 72 & 125 & 1.00 & 5.8 & B56 \\
\hline $146-012470$ & M & 11 & 38 & $5429_{-19}^{+22}$ & $3.9 \pm 0.1$ & $23245 \pm 47$ & 140 & 57 & 128 & 1.00 & - & B57 \\
\hline $146-012487$ & $\mathrm{NM}$ & 10 & 37 & $5058_{-14}^{+19}$ & $3.7 \pm 0.3$ & $-1191 \pm 44$ & 136 & 55 & 124 & 1.00 & - & B58 \\
\hline $147-012400$ & $\mathrm{NM}$ & 12 & 46 & $5040_{-13}^{+17}$ & $2.7 \pm 0.1$ & $97180 \pm 36$ & 125 & 45 & 116 & 1.00 & - & B62 \\
\hline $146-012635$ & M & 11 & 39 & $4862_{-13}^{+18}$ & $5.6 \pm 0.2$ & $22942 \pm 37$ & 122 & 56 & 109 & 1.00 & - & B63 \\
\hline 146-012496 & NM & 5 & 22 & $5111 \pm 33$ & $2.9 \pm 0.6$ & $20 \pm 50$ & 108 & 71 & 81 & 1.00 & - & B64 \\
\hline 147-012199 & NM & 12 & 59 & $5067_{-13}^{+17}$ & $2.7 \pm 0.1$ & $89852 \pm 30$ & 101 & 42 & 92 & 1.00 & 1.6 & B65 \\
\hline $146-012372$ & $\mathrm{NM}$ & 11 & 39 & $5194_{-16}^{+18}$ & $3.3 \pm 0.2$ & $77675 \pm 34$ & 97 & 56 & 80 & 0.98 & - & B66 \\
\hline
\end{tabular}

Note. RV Variables in the NGC 2516 field. The period is given for stars having more than six epochs and a peak (aside from the alias seen at $\sim 1 \mathrm{~d}$ ) exceeding 95 per cent significance. Derived values used our mean jitter level of $74 \mathrm{~m} \mathrm{~s}^{-1}$ for members and $58 \mathrm{~m} \mathrm{~s}^{-1}$ for non-members. Figure references are for Appendix B, which is available online.

Table 10. Variables in NGC 2422.

\begin{tabular}{|c|c|c|c|c|c|c|c|c|c|c|c|c|}
\hline UCAC4 & Flags & $N$ & $\overline{\mathrm{S} / \mathrm{N}}$ & $\begin{array}{l}T_{\text {eff }} \\
(\mathrm{K})\end{array}$ & $\begin{array}{c}v_{r} \sin (i) \\
\left(\mathrm{km} \mathrm{s}^{-1}\right)\end{array}$ & $\begin{array}{c}\mathrm{RV} \\
\left(\mathrm{m} \mathrm{s}^{-1}\right)\end{array}$ & $\begin{array}{c}\sigma_{\mathrm{obs}} \\
\left(\mathrm{m} \mathrm{s}^{-1}\right)\end{array}$ & $\begin{array}{c}\sigma_{\text {meas }} \\
\left(\mathrm{m} \mathrm{s}^{-1}\right)\end{array}$ & $\begin{array}{c}\sigma_{\text {stel }} \\
\left(\mathrm{m} \mathrm{s}^{-1}\right)\end{array}$ & $P_{\mathrm{v}}$ & $\begin{array}{l}\text { Period } \\
\text { (days) }\end{array}$ & Figure \\
\hline $378-036252$ & PMSB2 & 10 & 156 & $6324 \pm 73$ & $9.8 \pm 0.3$ & $47929 \pm 12207$ & 38107 & 95 & 38107 & 1.00 & 7.3 & B67 \\
\hline $378-036176$ & NMSB2 & 10 & 122 & $6102 \pm 148$ & $9.1 \pm 1.2$ & $4337 \pm 4762$ & 14124 & 110 & 14123 & 1.00 & 1.3 & B69 \\
\hline $379-035886$ & NMSB2 & 7 & 16 & $4999 \pm 44$ & $9.2 \pm 4.4$ & $26035 \pm 3938$ & 11504 & 190 & 11502 & 1.00 & - & B70 \\
\hline 379-035982 & MB & 8 & 45 & $4890_{-16}^{+21}$ & $14.1 \pm 3.5$ & $36530 \pm 2159$ & 5799 & 120 & 5798 & 1.00 & 2.0 & B73 \\
\hline $378-036328$ & MB & 10 & 116 & $6138_{-30}^{+38}$ & $7.9 \pm 0.2$ & $36928 \pm 1520$ & 5051 & 69 & 5050 & 1.00 & 27.7 & B74 \\
\hline 379-035884 & $\mathrm{NMB}$ & 10 & 124 & $6846_{-40}^{+52}$ & $19.0 \pm 0.3$ & $59672 \pm 1476$ & 4667 & 235 & 4661 & 1.00 & 559.4 & B75 \\
\hline $378-036136$ & MB & 10 & 27 & $5586 \pm 41$ & $9.0 \pm 0.2$ & $35631 \pm 1181$ & 3506 & 108 & 3505 & 1.00 & 543.7 & B76 \\
\hline 379-036005 & PMB & 10 & 86 & $6155_{-30}^{+38}$ & $19.1 \pm 0.8$ & $38391 \pm 426$ & 1386 & 207 & 1371 & 1.00 & - & B80 \\
\hline 379-036194 & NMB & 9 & 28 & $5425 \pm 29$ & $4.1 \pm 0.2$ & $106551 \pm 465$ & 1314 & 66 & 1312 & 1.00 & - & B81 \\
\hline $378-036137$ & NMB & 10 & 81 & $5503 \pm 24$ & $3.0 \pm 0.2$ & $119993 \pm 361$ & 1123 & 49 & 1122 & 1.00 & 230.3 & B82 \\
\hline $378-036814$ & MB & 10 & 49 & $5221_{-15}^{+19}$ & $6.9 \pm 0.2$ & $36322 \pm 335$ & 1023 & 68 & 1021 & 1.00 & 67.9 & B83 \\
\hline 377-035019 & $\mathrm{NMB}$ & 10 & 48 & $4555_{-14}^{+19}$ & $35.2 \pm 0.6$ & $32857 \pm 261$ & 805 & 238 & 769 & 1.00 & - & B84 \\
\hline $378-036277$ & NMB & 10 & 61 & $5382_{-20}^{+23}$ & $3.4 \pm 0.1$ & $27815 \pm 229$ & 721 & 38 & 720 & 1.00 & 1286.7 & B85 \\
\hline $378-036531$ & MB & 10 & 73 & $5672_{-20}^{+23}$ & $10.2 \pm 0.1$ & $37361 \pm 199$ & 635 & 78 & 630 & 1.00 & - & B86 \\
\hline 379-035545 & NMB & 10 & 23 & $4980_{-14}^{+19}$ & $4.3 \pm 0.2$ & $115336 \pm 141$ & 389 & 64 & 384 & 1.00 & - & B87 \\
\hline $379-035711$ & MB & 10 & 38 & $4656_{-14}^{+19}$ & $27.5 \pm 0.3$ & $35190 \pm 131$ & 378 & 184 & 330 & 1.00 & - & B88 \\
\hline $377-034937$ & NM & 10 & 75 & $6563_{-30}^{+38}$ & $17.0 \pm 0.2$ & $18602 \pm 103$ & 296 & 139 & 261 & 1.00 & - & B89 \\
\hline
\end{tabular}


Table 10 - continued

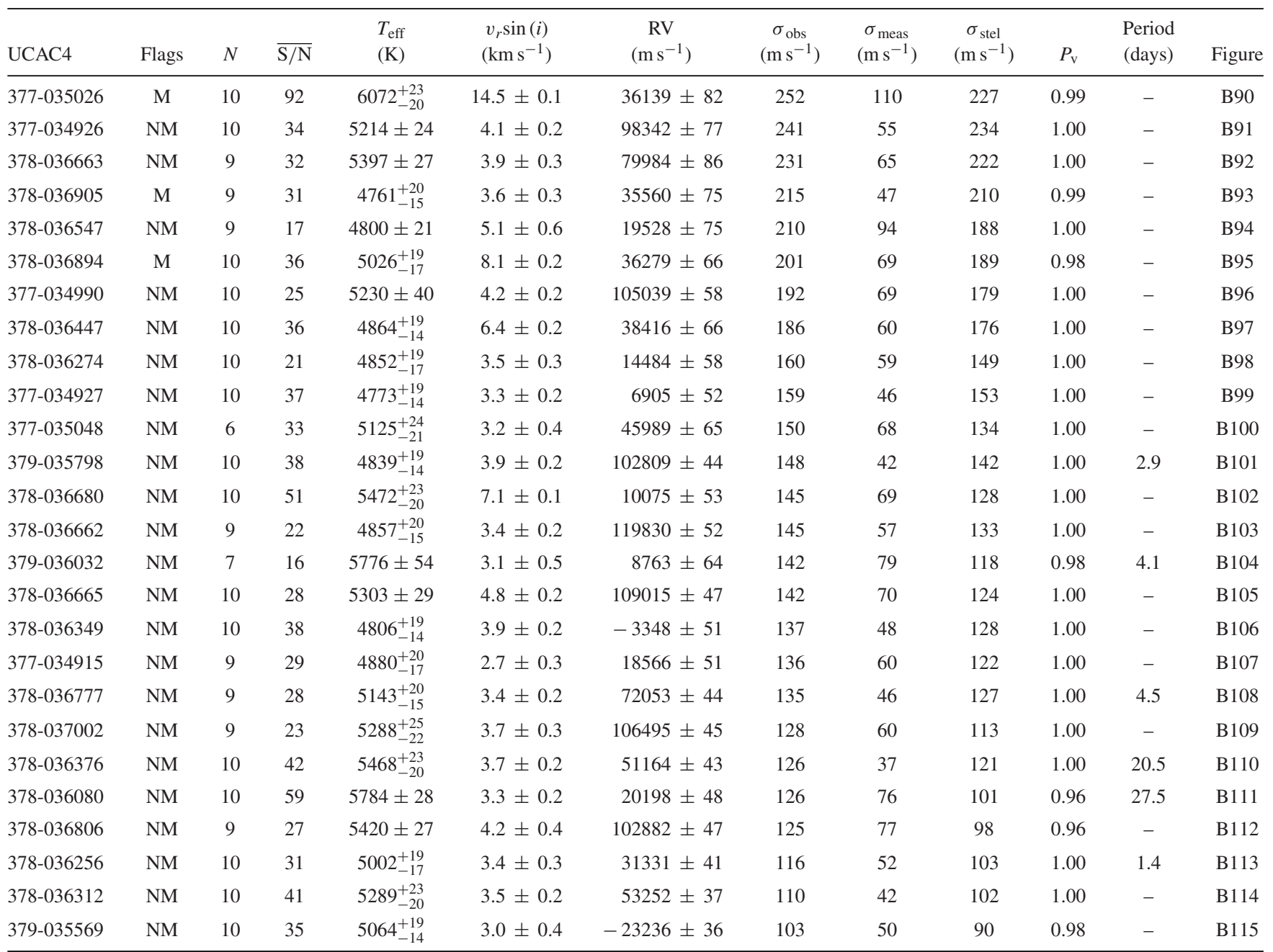

Note. RV variables in the NGC 2422 field. The period is given for stars having more than six epochs and a peak (aside from the alias seen at $\sim 1 \mathrm{~d}$ ) exceeding 95 per cent significance. Derived values used our mean jitter level of $138 \mathrm{~m} \mathrm{~s}^{-1}$ for members and $58 \mathrm{~m} \mathrm{~s}^{-1}$ for non-members. Figure references are for Appendix B, which is available online.

among the RV non-member non-variable stars in our NGC 2422 sample (Section 5.4).

We identified 52 stellar or brown dwarf RV binaries $\left(\sigma_{\text {stel }} \geq\right.$ $300 \mathrm{~m} \mathrm{~s}^{-1}$; see Section 4), 45 of which (17 in NGC 2516, 11 in NGC 2422 and 17 non-members) had not previously been identified as binaries. We were able to identify tentative periods for 38 of the RV binaries. The values are given in Tables 9 and 10. These periods suggest eight have lower mass limits consistent with brown dwarfs. We also reported the discovery of eight SB2s. Our sample of binaries is 99 per cent complete for $P \lesssim 8 \mathrm{yr}$.

We found an average stellar-activity-induced RV uncertainty of $74 \mathrm{~m} \mathrm{~s}^{-1}$ in NGC 2516 and $138 \mathrm{~m} \mathrm{~s}^{-1}$ in NGC 2422, broadly consistent with the known values for stellar jitter at ages of 141 and 73 Myr, respectively (Fig. 20). After accounting for this uncertainty, we identified 43 statistically significant low-amplitude $R V$ variables (16 in NGC 2516, 3 in NGC 2422 and 34 non-members). Nine (two in NGC 2516, one in NGC 2422 and six non-members) of these low-amplitude RV variables exhibited at least 95 per cent significant Lomb-Scargle periodogram peaks, all but one of which were consistent with a possible planetary companion. For RV non-variable stars in our sample, we are able to exclude companions more massive than about 8,12 and $24 \mathrm{M}_{\mathrm{Jup}}$ - on average - with 95 per cent confidence for orbits of 3,10 and $20 \mathrm{~d}$.

\section{ACKNOWLEDGEMENTS}

The authors gratefully acknowledge valuable discussions with Sam Quinn, Justin Cantrell, Colin Slater and Ian Roederer. JB acknowledges the support of the Netherlands Research School for Astronomy and the European Space Agency under European Space Research and Technology Centre contract 21114/07/NL/HBX. JB and $\mathrm{MM}$ acknowledge support from the National Science Foundation (NSF) astronomy and astrophysics research grant (AAG) 1312997 and the NSF/Major Research Instrumentation development grant 0923160. RW acknowledges support from NSF AAG 1517762. PyRAF is a product of the Space Telescope Science Institute, which is operated by the Association of Universities for Research in Astronomy for NASA. This research made use of ASTROPY, a community-developed core Python package for astronomy (Paunzen 2008). This research used the WEBDA database, operated at the Department of Theoretical Physics and Astrophysics of Masaryk University. 


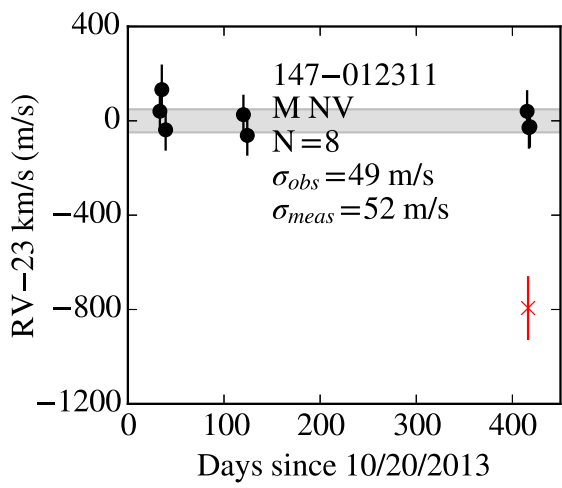

(a)

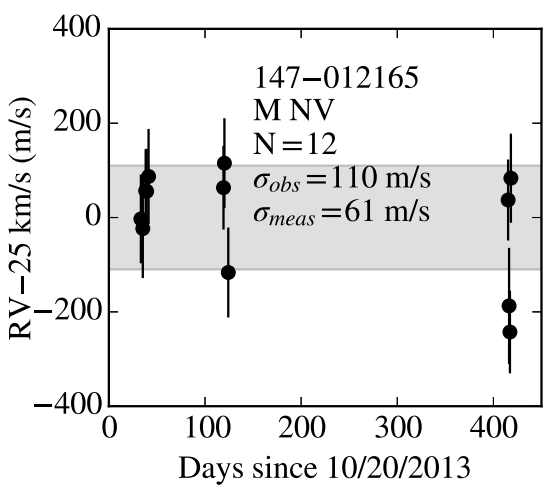

(d)

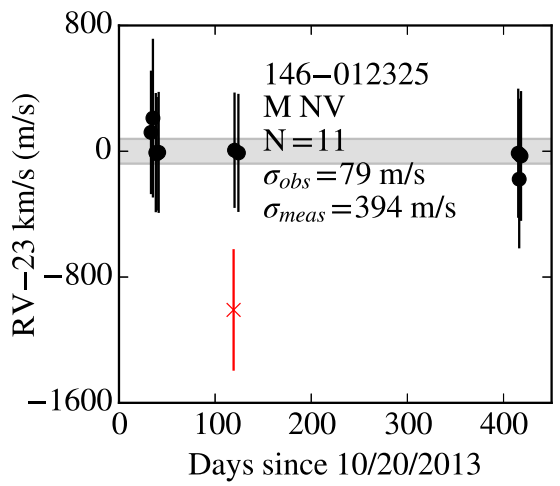

(b)

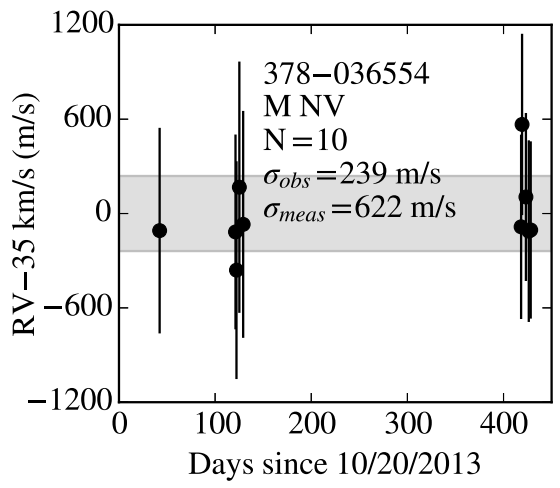

(e)

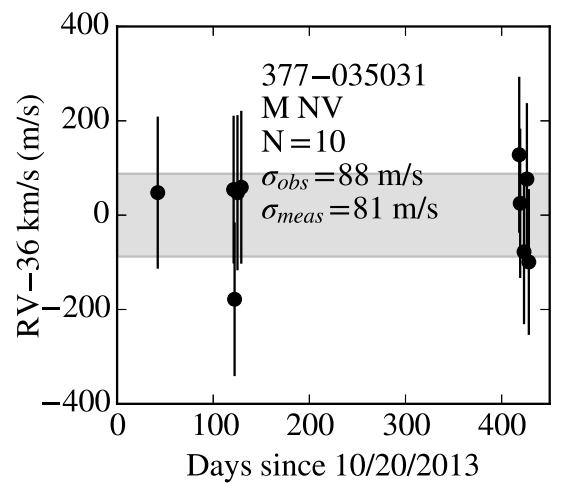

(c)

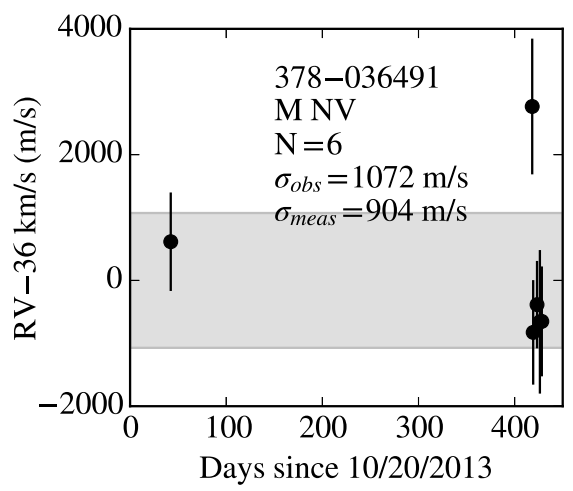

(f)

Figure 24. RV time series for a selection of RV non-variable stars in our sample. Properties are provided in Table 11 . IDs starting with 1 are in NGC 2516 and 3 in NGC 2422. The grey band is of width $2 \sigma_{\text {obs }}$. Red X's are epochs excluded as $>3 \sigma_{\text {obs }}$ outliers. Membership and variability codes are shown for each star directly beneath the ID and are as described in the text. Errors shown include the appropriate $\sigma_{\text {jitter }}$.

Table 11. Selected RV non-variables in NGC 2516 and NGC 2422.

\begin{tabular}{|c|c|c|c|c|c|c|c|c|c|c|}
\hline ID & $N$ & $\mathrm{~S} / \mathrm{N}$ & $\begin{array}{l}T_{\text {eff }} \\
(\mathrm{K})\end{array}$ & $\begin{array}{c}v_{r} \sin (i) \\
\left(\mathrm{km} \mathrm{s}^{-1}\right)\end{array}$ & $\begin{array}{c}\mathrm{RV} \\
\left(\mathrm{m} \mathrm{s}^{-1}\right)\end{array}$ & $\begin{array}{c}\sigma_{\mathrm{obs}} \\
\left(\mathrm{m} \mathrm{s}^{-1}\right)\end{array}$ & $\begin{array}{c}\sigma_{\text {meas }} \\
\left(\mathrm{m} \mathrm{s}^{-1}\right)\end{array}$ & $P_{\mathrm{v}}$ & Code & Plot \\
\hline $147-012311$ & 8 & 31 & $5073_{-16}^{+21}$ & $3.8 \pm 0.2$ & $22877 \pm 25$ & 49 & 52 & 0.11 & M NV & Fig. $24 \mathrm{a}$ \\
\hline $377-035031$ & 10 & 63 & $5441 \pm 26$ & $9.8 \pm 0.1$ & $35610 \pm 38$ & 88 & 81 & 0.04 & M NV & Fig. $24 \mathrm{c}$ \\
\hline $147-012165$ & 12 & 31 & $5093_{-13}^{+17}$ & $4.5 \pm 0.2$ & $25188 \pm 38$ & 110 & 61 & 0.86 & M NV & Fig. $24 \mathrm{~d}$ \\
\hline
\end{tabular}

Note. Properties for a selection of RV non-variable members stars in our sample. RV time series plots are shown in Fig. 24. IDs starting with 1 are in NGC 2516 and 3 in NGC 2422 .

\section{REFERENCES}

Bailey John I., III, White R. J., Blake C. H., Charbonneau D., Barman T. S., Tanner A. M., Torres G., 2012, ApJ, 749, 16

Bailey J. I., III, Mateo M., White R. J., Shectman S. A., Crane J. D., Olszewski E. W., 2016, AJ, 152, 9 (B16)

Boss A. P., 1997, Science, 276, 1836

Brucalassi A. et al., 2014, A\&A, 561, L9

Butler R. P., Marcy G. W., Williams E., McCarthy C., Dosanjh P., Vogt S. S., 1996, PASP, 108, 500

Casagrande L., Ramírez I., Meléndez J., Bessell M., Asplund M., 2010, A\&A, 512, 54

Damiani F., Flaccomio E., Micela G., Sciortino S., Harnden F. R., Jr, Murray S. S., Wolk S. J., Jeffries R. D., 2003, ApJ, 588, 1009 (D03)
Duchêne G., Kraus A., 2013, ARA\&A, 51, 269 (DK13)

Fabrycky D., Tremaine S., 2007, ApJ, 669, 1298

Goldreich P., Tremaine S., 1980, ApJ, 241, 425

Herbig G. H., Soderblom D. R., 1982, ApJ, 252, 610

Howell S. B. et al., 2014, PASP, 126, 398

Husser T. O., Wende-von Berg S., Dreizler S., Homeier D., Reiners A., Barman T., Hauschildt P. H., 2013, A\&A, 553, A6

Jeffries R. D., Thurston M. R., Hambly N. C., 2001, A\&A, 375, 863 (J01) Johnson J. A., Howard A. W., Bowler B. P., Henry G. W., Marcy G. W., Wright J. T., Fischer D. A., Isaacson H., 2010, PASP, 122, 701

Jurić M., Tremaine S., 2008, ApJ, 686, 603

Kharchenko N. V., Piskunov A. E., R"oser S., Schilbach E., Scholz R. D., 2005, A\&A, 438, 1163 
Lagrange A.-M., Meunier N., Chauvin G., Sterzik M., Galland F., Lo Curto G., Rameau J., Sosnowska D., 2013, A\&A, 559, A83

Lin D. N. C., Bodenheimer P., Richardson D. C., 1996, Nature, 380, 606

Loktin A. V., Gerasimenko T. P., Malysheva L. K., 2001, Astronom. Astrophys. Trans., 20, 607

Mateo M., Bailey J. I., Crane J., Shectman S., Thompson I., Roederer I., Bigelow B., Gunnels S., 2012, Proc. SPIE, 8446, 84464Y

Meibom S. et al., 2013, Nature, 499, 55

Meschiari S., Wolf A. S., Rivera E., Laughlin G., Vogt S., Butler P., 2012, Astrophysics Source Code Library, ascl:1210.018

Meynet G., Mermilliod J.-C., Maeder A., 1993, A\&AS, 98, 477

Mizuno H., 1980, Prog. Theor. Phys., 64, 544

Nissen P. E., 1988, A\&A, 199, 146

Paulson D. B., Yelda S., 2006, PASP, 118, 706

Paulson D. B., Cochran W. D., Hatzes A. P., 2004, AJ, 127, 3579

Paunzen E., 2008, CoSkS, 38, 435

Pillitteri I., Micela G., Damiani F., Sciortino S., 2006, A\&A, 450, 993

Prisinzano L., Micela G., Sciortino S., Favata F., 2003, A\&A, 404, 927 (P03)

Queloz D., Allain S., Mermilliod J.-C., Bouvier J., Mayor M., 1998, A\&A, 335,183

Quinn S. N. et al., 2012, ApJ, 756, L33

Quinn S. N. et al., 2014, ApJ, 787, 27

Rasio F. A., Ford E. B., 1996, Science, 274, 954

Robin A. C., Reylé C., Derrière S., Picaud S., 2003, A\&A, 409, 523

Schlafly E. F., Finkbeiner D. P., 2011, ApJ, 737, 103

Slater C. T. et al., 2014, ApJ, 791, 9

Soubiran C., Jasniewicz G., Chemin L., Crifo F., Udry S., Hestroffer D., Katz D., 2013, A\&A, 552, A64

Sung H., Bessell M. S., Lee B.-W., Lee S.-G., 2002, AJ, 123, 290
Terndrup D. M., Pinsonneault M., Jeffries R. D., Ford A., Stauffer J. R., Sills A., 2002, ApJ, 576, 950

Torres G., Andersen J., Giménez A., 2010, A\&AR, 18, 67

Udry S., Santos N. C., 2007, ARA\&A, 45, 397

van Dokkum P. G., 2001, PASP, 113, 1420

VanderPlas J. T., Ivezić Ž., 2015, ApJ, 812, 18

Walker M. G., Olszewski E. W., Mateo M., 2015, MNRAS, 448, 2717

Wallace L., Hinkle K. H., Livingston W. C., Davis S. P., 2011, ApJS, 195, 6

Winn J. N., Fabrycky D. C., 2015, ARA\&A, 53, 409

Wolk S. J. et al., 2004, ApJ, 606, 466

Wright J. T., Marcy G. W., Howard A. W., Johnson J. A., Morton T. D., Fischer D. A., 2012, ApJ, 753, 160

Zacharias N., Finch C. T., Girard T. M., Henden A., Bartlett J. L., Monet D. G., Zacharias M. I., 2013, AJ, 145, 44

\section{SUPPORTING INFORMATION}

Supplementary data are available at MNRAS online.

Please note: Oxford University Press is not responsible for the content or functionality of any supporting materials supplied by the authors. Any queries (other than missing material) should be directed to the corresponding author for the article.

This paper has been typeset from a $\mathrm{T}_{\mathrm{E}} \mathrm{X} / \mathrm{L} \mathrm{T}_{\mathrm{E}} \mathrm{X}$ file prepared by the author. 